\title{
On the stable eigenvalues of perturbed anharmonic oscillators in dimension two
}

\author{
Dario Bambusi, Beatrice Langellał, Marc Rouveyrolł
}

\begin{abstract}
We study the asymptotic behavior of the spectrum of a quantum system which is a perturbation of a spherically symmetric anharmonic oscillator in dimension 2. We prove that a large part of its eigenvalues can be obtained by Bohr-Sommerfeld quantization rule applied to the normal form Hamiltonian and also admit an asymptotic expansion at infinity. The proof is based on the generalization to the present context of the normal form approach developed in BLM20b] (see also [PS10]) for the particular case of $\mathbb{T}^{d}$.
\end{abstract}

Keywords: Schrödinger operator, normal form, pseudo differential operators

MSC 2010: 37K10, 35Q55

\section{Contents}

1 Introduction 2

2 Main result 5

2.1 Symbols ....................... 5

2.2 The integrable case . . . . . . . . . . . . . . . . 7

2.3 Main result . . . . . . . . . . . . . . . . 8

${ }^{*}$ Dipartimento di Matematica, Università degli Studi di Milano, Via Saldini 50, I-20133 Milano. Email: dario.bambusi@unimi.it

${ }^{\dagger}$ Dipartimento di Matematica, Università degli Studi di Milano, Via Saldini 50, I-20133 Milano. Email: beatrice.langella@unimi.it

${ }^{\ddagger}$ DER de mathématiques, ENS Paris-Saclay, 4 Avenue des Sciences, 91190 Gif-surYvette. Email: marc.rouveyrol@ens-paris-saclay.fr 
4 Properties of $h_{0}$

5 Symbols, Fourier expansions and Pseudo-differential calculus $\quad 14$

5.1 A class of symbols . . . . . . . . . . . . . . . . 14

5.2 Flow of the actions . . . . . . . . . . . . . 15

5.3 Fourier expansion and summable symbols . . . . . . . . . . 17

6 The normal form lemma 21

7 Spectral result $\quad 28$

8 Cardinality estimates 33

8.1 Nondegenerate homogeneous frequency maps . . . . . . . . . 32

8.2 Nondegeneracy of the frequency map of the anharmonic oscil-

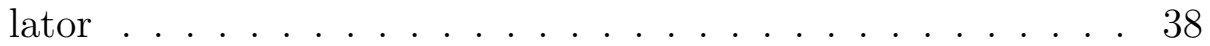

A Proof of Lemma 4.5 40

B Proof of Lemma 5.12

\section{Introduction}

In this paper we study the asymptotic behavior of the spectrum of the quantum system

$$
\begin{aligned}
& H:=H_{0}+V(x,-\mathrm{i} \nabla), \quad x \in \mathbb{R}^{2}, \\
& H_{0}:=-\frac{1}{2} \Delta+\frac{\|x\|^{2 \ell}}{2 \ell}, \quad \ell \in \mathbb{N}^{*}, \quad \ell \geq 2, \\
& \|x\|^{2 \ell}:=\left(x_{1}^{2}+x_{2}^{2}\right)^{\ell},
\end{aligned}
$$

where $V(x,-\mathrm{i} \nabla)$ is a self-adjoint pseudo-differential operator of order smaller than $H_{0}$. The idea is to consider the system (1.1) as a perturbation of the quantum integrable system 1.2 and to apply the quantization of normal form theory as developed in [BLM20b] (see also [Par08, PS10, PS12], [BLM20c] in order to obtain an asymptotic expansion of a large part of the eigenvalues of (1.1).

To describe our result, consider first the integrable system $H_{0}$; it was proved by Charbonnel [Cha86, Cha83b] that a large part of its spectrum can be constructed through Bohr-Sommerfeld rule. Precisely there exists a 
function $\tilde{h}_{0}$ with the property that $\tilde{h}_{0}(\mathrm{a})$ is asymptotic to an eigenvalue of $H_{0}$ for all a's in a subset $C$ of the lattice $\mathbb{Z}^{2}+\kappa$ with $\kappa \in \mathbb{R}^{2}$ fixed. The function $\tilde{h}_{0}$ is a perturbation of the classical Hamiltonian $h_{0}$ written in terms of action angle variables.

Here we prove that there exists a subset $\Omega \subset \mathrm{C}$ of density 1 in $\mathrm{C}$ and a function $\tilde{h}$ s.t. for all $\mathrm{a} \in \Omega$ there exists an eigenvalue $\lambda_{\mathrm{a}}$ of 1.1 with

$$
\lambda_{\mathrm{a}} \sim \tilde{h}(\mathrm{a}) .
$$

Furthermore, $\tilde{h}$ is a perturbation of $\tilde{h}_{0}$ and admits an asymptotic expansion in powers of $\|a\|^{-1}$.

Results of this kind are completely standard in the case of quantum oscillators in dimension 1 , but we are not aware of a single result of this kind in dimension at least 2 (see however [Roy07, HSVN07] for related results).

The present paper can be considered as a continuation of the works [BLM20b, BLM20c, BLM20a], in particular of [BLM20b], in which we studied the stable eigenvalues of Schrödinger operators on $\mathbb{T}^{d}$. The idea of [BLM20b] consists in working on the symbol of the quantum operator in order to conjugate $H$ to a system in "quantum normal form". Now, in classical Hamiltonian mechanics, it is well known that the normal form of a system is particularly simple only in the regions of the phase space where the frequencies are non resonant. A similar property holds also for the quantum system and it turns out that the eigenvalues which correspond to the classically nonresonant region can be obtained by Bohr-Sommerfeld quantization rule, and furthermore admit an asymptotic expansion in powers of $\lambda^{-1}$.

The starting point of the present paper is the idea that all the techniques developed in [BLM20b, BLM20c, BLM20a, PS10] should apply to any quantum system which is a perturbation of an integrable quantum system. However there are several technical difficulties to overcome in order to actually transform such a heuristic statement into a theorem and, in order to avoid the risk of getting empty results, we decided to start our investigation from a concrete model which has some interest in itself, namely the quantum anharmonic oscillator. The main aim of this paper is to develop the tools needed to apply the ideas of [BLM20b, BLM20c, BLM20a] to general perturbations of quantum integrable systems, and to start to deduce some consequences.

The main technical difficulties with respect to [BLM20b] are of four kinds:

(1) find a class of symbols suitable for the normal form construction and deal with it

(2) generalize the construction of [BLM20b] to the case where the frequencies do not coincide with the actions, but are just a function of the actions which is poorly known 
(3) verify that all the needed nonresonance properties are satisfied in the concrete case of the quantum anharmonic oscillator

(4) use a suitable version of functional calculus in order to obtain spectral properties of operators in quantum normal form.

Concerning (1), we use a class of symbols which is a small modification of the class used by Helffer and Robert in [HR82b, HR82a]. However, it turns out that such a class of symbols behaves badly under Fourier expansion, so we use a trick from classical normal form theory in order to avoid to re-expand symbols in Fourier series at each step of the iteration. Concerning (2), we use here the remarkable fact that the actions turn out to be quasi-homogeneous functions of the phase space variables. Furthermore the Hamiltonian and the frequencies turn out to be homogeneous functions of the actions. Concerning (3), we first prove that the actions are analytic functions of the phase space variables which are globally defined. We do it by a direct computation, but the computation is enlightened by the general theory of action angle variables in the form of [BF16]. Then we have to verify that the subset of the phase space in which the frequencies are nonresonant has large measure. This is done using tools from degenerate KAM theory (see [Rüs01, BBM11]) and homogeneity of the frequency map. We also use some results from [FK04, BF17] (see also [BFS18]). The point (4) is solved using the same ideas developed by [Cha86.

As we mentioned above, our technique is a generalization of a technique introduced on $\mathbb{T}^{d}$, so we conclude this introduction by recalling the results which are known in that situation and that we hope to extend to more general situations in the future.

In the case of the Schrödinger operator on $\mathbb{T}^{d}$ it was shown by FKT90, Fri90] (see also [Vel15, Kar96, PS10]) that most of the eigenvalues $\lambda$ of the Laplacian are stable under perturbation, in the sense that all eigenvalues bifurcating from them admit a full asymptotic expansion in powers of $\lambda^{-1}$. On the contrary (see [FKT91]) some eigenvalues are unstable (in the terminology of [FKT91]), and in particular they do not admit such an asymptotic expansion. In BLM20b] the stable eigenvalues were recognized to correspond to the nonresonant regions of the classical phase space, and in BLM20c we proved that the unstable eigenvalues can be obtained as eigenvalues of a Schrödinger operator on a lower dimensional torus. Finally in BLM20a we used the results of [BLM20c] to study the case of time dependent potentials and to prove a $\langle t\rangle^{\epsilon}$ estimate on the growth of Sobolev norms of the solutions of the time dependent Schrödinger equation. We plan to investigate analogous problems for the anharmonic oscillator in the future. 
Acknowledgments First of all we thank Didier Robert for pointing to our attention the papers by Charbonnel, then we thank Francesco Fassò for pointing to our attention the paper [BF16] and San Vũ Ngọc for several suggestions on quantum action angle coordinates. During the preparation of this work we also had several discussions with Alberto Maspero, that we warmly thank.

We thank the Italian Gruppo Nazionale di Fisica Matematica of INDAM for the support.

\section{Main result}

\section{$2.1 \quad$ Symbols}

As usual, we define a scale of Sobolev like spaces adapted to our situation. For all $s \geq 0$ we define $\mathcal{H}^{s}=D\left(H_{0}^{s \frac{\ell+1}{2 \ell}}\right.$ ) (where $D(\cdot)$ is the domain of an operator), while if $s<0$ we define $\mathcal{H}^{s}=\left(\mathcal{H}^{-s}\right)^{\prime}$, where $V^{\prime}$ is the dual space to $V$ with respect to $\mathcal{H}^{0}=L^{2}\left(\mathbb{R}^{2}\right)$. We consider such spaces endowed with the natural norms $\|\psi\|_{s}:=\left\|H_{0}^{s \frac{\ell+1}{2 \ell}} \psi\right\|_{L^{2}}$.

We will denote by $\mathcal{B}\left(\mathcal{H}^{s}, \mathcal{H}^{s^{\prime}}\right)$ the space of bounded linear operators from $\mathcal{H}^{s}$ to $\mathcal{H}^{s^{\prime}}$, and, given an operator $A \in \mathcal{B}\left(\mathcal{H}^{s}, \mathcal{H}^{s^{\prime}}\right)$, we will denote by $\|A\|_{s, s^{\prime}}$ its norm in $\mathcal{B}\left(\mathcal{H}^{s}, \mathcal{H}^{s^{\prime}}\right)$.

Definition 2.1. Given $N \in \mathbb{R}^{+}$we say that an operator $R$ is smoothing of order $N$ if $R \in \bigcap_{s \in \mathbb{R}} \mathcal{B}\left(\mathcal{H}^{s}, \mathcal{H}^{s+N}\right)$.

If $R$ is smoothing of order $N$ for any $N \in \mathbb{R}^{+}$, we say $R$ is an (infinitely) smoothing operator.

Following [HR82b], we define now a first class of symbols. Denote

$$
\mathrm{k}_{0}(x, \xi):=\left(1+\|x\|^{2 \ell}+\|\xi\|^{2}\right)^{\frac{\ell+1}{2 \ell}}, \quad\|x\|:=\sqrt{x_{1}^{2}+x_{2}^{2}}
$$

Definition 2.2. A function $f=f(x, \xi)$ will be called a symbol of order $m \in \mathbb{R}$ if $f \in C^{\infty}\left(\mathbb{R}^{4}\right)$ and $\forall \alpha, \beta \in \mathbb{N}^{2}$, there exists $C_{\alpha, \beta}>0$ s.t.

$$
\left|\partial_{\xi}^{\alpha} \partial_{x}^{\beta} f(x, \xi)\right| \leq C_{\alpha, \beta} \mathrm{k}_{0}(x, \xi)^{m-\frac{|\beta|+\ell|\alpha|}{\ell+1}},
$$

where $|\alpha|:=\left|\alpha_{1}\right|+\left|\alpha_{2}\right|$, and similarly for $|\beta|$. In this case we will write $f \in S_{H R}^{m}$. 
Definition 2.3. To any symbol $f \in S_{H R}^{m}, m \in \mathbb{R}$, we associate an operator $O p^{w}(f)$ acting on the scale $\left\{\mathcal{H}^{s}\right\}_{s \in \mathbb{R}}$ as the Weyl quantization of $f$, namely:

$$
\left[O p^{w}(f) \psi\right](x)=\frac{1}{4 \pi^{2}} \int_{\mathbb{R}^{2}} \int_{\mathbb{R}^{2}} f\left(\frac{x+y}{2}, \xi\right) \psi(y) e^{\mathrm{i}(x-y) \xi} d y d \xi
$$

Conversely, if there exist a symbol $f \in S_{H R}^{m}$ s.t.

$$
F=O p^{w}(f),
$$

we say that $F$ is a pseudo-differential operator of order $m$, and write $F \in$ $O P S_{H R}^{m}$.

We also need to deal with symbols which are function of the actions only.

Definition 2.4. Given $\varsigma>0, m \in \mathbb{R}$, we define the class of classical symbols $S_{C}^{m, \varsigma}$ as the set of all the functions $f \in \mathbb{C}^{\infty}\left(\mathbb{R}^{2}\right)$ such that for any $\alpha \in \mathbb{N}^{2}$, there exists $C_{\alpha}$ s.t.

$$
\left|\partial_{a}^{\alpha} g(a)\right| \leq C_{\alpha}\langle a\rangle^{m-\varsigma|\alpha|},
$$

where, as usual, $\langle a\rangle:=\sqrt{1+\|a\|^{2}}$.

Definition 2.5. Given a sequence of symbols $\left\{f_{j}\right\}_{j \geq 0}$ with $f_{j} \in S_{C}^{m-\rho j, \varsigma}$ for some $m \in \mathbb{R}$ and $\rho, \varsigma>0$, and a function $f(a)$, we write

$$
f \sim \sum_{j} f_{j}
$$

if for any $N \in \mathbb{N}$ there exists $C_{N}$ s.t.

$$
\left|f(a)-\sum_{j=0}^{N} f_{j}(a)\right| \leq C_{N}\langle a\rangle^{m-(N+1) \rho} .
$$

We will use similar notations for all different classes of symbols we will meet in the following. We finally fix some further notation: given two quantities $a, b \in \mathbb{R}$, we write $a \lesssim b$ if there exists a positive constant $C$, independent of all the relevant quantities, such that $a \leq C b$. We will occasionally write $a \lesssim_{s} b$ if the constant $C$ depends on the parameter $s$. We will also write $a \simeq b$ if $a \lesssim b$ and $b \lesssim a$. 


\subsection{The integrable case}

We present here the results of Charbonnel [Cha86] on the spectrum of $H_{0}$, in a form suitable for our developments.

Consider the classical Hamiltonian system

$$
h_{0}(x, \xi):=\frac{\|\xi\|^{2}}{2}+\frac{\|x\|^{2 \ell}}{2 \ell} .
$$

whose quantization is $H_{0}$. We introduce now action variables $a_{1}, a_{2}$ for $h_{0}$; it turns out (see Lemma 4.5 below) that their range is the cone

$$
\Pi:=\left\{a \in \mathbb{R}^{2} ; a_{1} \geq 0 \text { if } a_{2} \geq 0, \quad a_{1} \geq\left|a_{2}\right| \text { if } a_{2}<0\right\} ;
$$

we fix once for all an open cone $\mathcal{C}$ such that $\overline{\mathcal{C}} \backslash\{0\}$ is contained in the interior of $\Pi$.

Consider now the operators $A_{1}, A_{2}$ obtained by Weyl quantization of the actions $a_{1}, a_{2}$. Since $\left[A_{1} ; A_{2}\right]=0$ (as it immediately follows from the fact that $a_{2}$ is the angular momentum, which is a quadratic polynomial in $x, \xi$ ), one can consider their joint spectrum. Precisely there exist two diverging sequences $\lambda_{\mathrm{a}_{1}}^{(1)}$ and $\lambda_{\mathrm{a}_{2}}^{(2)}$ and a basis of $L^{2}$ formed by joint eigenfunctions $\psi_{\mathrm{a}}$, $\mathrm{a} \equiv\left(\mathrm{a}_{1}, \mathrm{a}_{2}\right)$ :

$$
A_{1} \psi_{\mathrm{a}}=\lambda_{\mathrm{a}_{1}}^{(1)} \psi_{\mathrm{a}}, \quad A_{2} \psi_{\mathrm{a}}=\lambda_{\mathrm{a}_{2}}^{(2)} \psi_{\mathrm{a}}
$$

Then

$$
\Lambda^{A}:=\left\{\left(\lambda_{\mathrm{a}_{1}}^{(1)}, \lambda_{\mathrm{a}_{2}}^{(2)}\right)\right\}
$$

is called the joint spectrum of $A_{1}$ and $A_{2}$. The following theorem is essentially Theorem 2.4 of [Cha86]

Theorem 2.6. There exist $\kappa \in \mathbb{R}^{2}$ and $C_{0}$ with the following properties:

$$
\Lambda^{A} \cap \mathcal{C} \subset \bigcup_{\mathrm{a} \in \mathbb{Z}^{2}+\kappa} B_{\left(\frac{C_{0}}{\|\mathrm{a}\|}\right)}(\mathrm{a})
$$

where $B_{R}(\mathrm{a})$ is the closed ball in $\mathbb{R}^{2}$ of radius $R$ and center $\mathrm{a}$.

Furthermore, for $\mathrm{a} \in\left(\mathbb{Z}^{2}+\kappa\right) \cap \mathcal{C}$ large enough

$$
\sharp\left(\Lambda^{A} \cap B_{\left(\frac{C_{0}}{\|\mathrm{a}\|}\right)}(\mathrm{a})\right)=1 \text {. }
$$

Actually, from functional calculus (see Cha83a]) one can deduce an asymptotic expansion of the eigenvalues. 
Theorem 2.7. Let

$$
\mathrm{C}:=\left(\mathbb{Z}^{2}+\kappa\right) \cap \mathcal{C},
$$

with $\kappa$ as in Theorem 2.6. There exists a sequence $\tilde{h}_{0, j} \in S_{C}^{\frac{2 \ell}{\ell+1}-j, 1}, j \geq 0$, of symbols with the following property: for any $\mathrm{a} \in \mathrm{C}$ large enough, there exists a unique eigenvalue $\lambda_{\mathrm{a}}^{(0)}$ of $H_{0}$ fulfilling

$$
\lambda_{\mathrm{a}}^{(0)}=\tilde{h}_{0}(\mathrm{a}) \sim \sum_{j \geq 0} \tilde{h}_{0, j}(\mathrm{a}) .
$$

Furthermore $\tilde{h}_{0,0}$ is the Hamiltonian $h_{0}$ written in action angle variables.

\subsection{Main result}

We are now ready to state our main result, which is the following Theorem.

Theorem 2.8. Consider the operator

$$
H:=H_{0}+V
$$

with $V \in O P S_{H R}^{m}$ and

$$
m<\frac{2 \ell}{\ell+1}
$$

define

$$
M:=\frac{\ell-1}{\ell+1}, \quad \mathfrak{e}:=\frac{2 \ell}{\ell+1}-m, \quad \delta_{0}:=M-\min \left\{\frac{1}{\ell+1}, \frac{\mathfrak{e}}{3}, \frac{2}{7}\right\},
$$

then there exists $\mu_{0}>0$ such that for any choice of the parameters $\delta$ and $\epsilon$ satisfying

$$
\delta_{0}<\delta<M, \quad 0<\epsilon<\frac{M-\delta}{2 \mu_{0}}
$$

define

$$
\varsigma:=1-(M-\delta), \quad \rho:=\min \{\mathfrak{e}-3(M-\delta), 2-7(M-\delta)\},
$$

then the following holds.

There exists a sequence of symbols $\left\{\tilde{z}_{j}\right\}_{j \in \mathbb{N}}$ with $\tilde{z}_{j} \in S_{C}^{m-j \rho, \varsigma}$ and a set $\Omega \subset \mathrm{C}$ such that

1. $\Omega$ has density one at infinity in $\mathrm{C}$, more precisely, denoting $\forall R>0$ $B_{R}:=B_{R}(0)$, one has

$$
1-\frac{\sharp\left(\Omega \cap B_{R}\right)}{\sharp\left(B_{R} \cap \mathrm{C}\right)}=\mathcal{O}\left(R^{-\frac{(M-\delta)}{\mu_{0}}+2 \epsilon}\right) \quad \text { as } R \rightarrow+\infty \text {; }
$$


2. for any $\mathrm{a} \in \Omega$ there exists an eigenvalue $\lambda_{\mathrm{a}}$ of 2.14 which admits the asymptotic expansion

$$
\lambda_{\mathrm{a}} \sim \tilde{h}_{0}(\mathrm{a})+\sum_{j \geq 0} \tilde{z}_{j}(\mathrm{a}), \quad \mathrm{a} \in \Omega
$$

where $\tilde{h}_{0}(\mathrm{a})$ is the function in 2.13 .

Remark 2.9. An example of a perturbation fulfilling the assumptions of Theorem 2.8 is the Weyl quantization of

$$
v(x, \xi):=\sum_{|\alpha|+\ell|\beta|<2 \ell} c_{\alpha, \beta} x^{\alpha} \xi^{\beta}
$$

where

$$
x^{\alpha}:=x_{1}^{\alpha_{1}} x_{2}^{\alpha_{2}}, \quad \xi^{\beta}:=\xi_{1}^{\beta_{1}} \xi_{2}^{\beta_{2}} .
$$

The rest of the paper is devoted to the proof of Theorem 2.8 .

\section{Scheme of the proof}

The idea of the proof is exactly the same of [BLM20b] (see also [PS10]). We now recall it in order to give a road map to the reader. The idea is to perform a "semiclassical normal form".

Consider first the classical Hamiltonian

$$
h:=h_{0}+V,
$$

with $h_{0}$ integrable. As already anticipated we denote by $a=\left(a_{1}, a_{2}\right)$ the classical actions of $h_{0}$. Suppose for a while to introduce action angle variables, so that $h_{0}$ turns out to be a function of the actions only.

We are interested in studying the system in the region of large $a$. In such a region, $V$ can be considered as a perturbation of $h_{0}$. Thus we develop a perturbation theory in which the order of perturbation is given by inverse powers of $\|a\|$.

The classical normal form procedure consists in looking for an auxiliary Hamiltonian function $g$ s.t. the corresponding time 1 flow $\phi_{g}^{1}$ (namely the time one flow of the corresponding Hamiltonian vector field) conjugates $h$ to a new Hamiltonian $h \circ \phi_{g}^{1}$ which, up to lower orders, is a function of the actions only.

By a formal computation one has (see below)

$$
h \circ \phi_{g}=h_{0}+\left\{g, h_{0}\right\}+V+\text { lower order terms }
$$


where $\{\cdot, \cdot\}$ are the Poisson brackets. Then, the main point is to determine $g$ in such a way that

$$
\left\{g, h_{0}\right\}+V=\text { function of } a \text { only . }
$$

However, this can be done only in the nonresonant regions. To explain the situation pass to action angle variables $(a, \varphi)$ and expand $V$ in Fourier series in the angles: one has

$$
V(a, \varphi)=\sum_{k \in \mathbb{Z}^{2}} V_{k}(a) e^{\mathrm{i} k \cdot \varphi}
$$

and, defining the frequencies as

$$
\omega_{i}(a):=\frac{\partial h_{0}}{\partial a_{i}}(a)
$$

one gets

$$
\left\{h_{0} ; \cdot\right\}=-\omega \cdot \frac{\partial}{\partial \varphi},
$$

so that one is led to try to define the function $g$ as

$$
\sum_{k \neq 0} \frac{V_{k}(a)}{\mathrm{i} \omega(a) \cdot k} e^{\mathrm{i} k \cdot \varphi}
$$

which of course is ill defined in the resonant region where the denominators vanish. To overcome this problem we introduce a cutoff to localize outside the resonant regions. It turns out that a suitable cutoff can be defined as follows: fix once for all a function $\chi \in C^{\infty}(\mathbb{R})$ which is equal to 1 in $B_{1 / 2}(0)$ and vanishes outside $B_{1}(0)$, define

$$
\chi_{k}(a):=\chi\left(\frac{\omega \cdot k}{\|k\|\|a\|^{\delta}}\right), \quad \tilde{\chi}_{k}(a):=\chi\left(\frac{\|k\|}{\|a\|^{\epsilon}}\right),
$$

and put

$$
g(a, \varphi):=\sum_{k \neq 0} \frac{V_{k}(a)}{\mathrm{i} \omega(a) \cdot k}\left(1-\chi_{k}(a)\right) \tilde{\chi}_{k}(a) e^{\mathrm{i} k \cdot \varphi},
$$

which can be used to play the game it was designed for. By doing this and iterating the construction one conjugates the system to a normal form part, which in the nonresonant regions depends only on the actions plus a remainder which decreases at infinity as fast as one wants.

Now the point is to quantize this procedure. This was done in [BLM20b] for the case of the Schrödinger operator on the torus $\mathbb{T}^{d}$. The key remark 
is that, by Egorov theorem, one can simply quantize the transformation given by the Hamiltonian flow of $g$ and this gives a unitary transformation that conjugates $H$ to a "quantum normal form" "1. However the situation of [BLM20b] (and also of [Par08, PS10, PS12]) was quite simplified by the fact that the Laplacian on $\mathbb{T}^{d}$ is the quantization of $h_{0}:=\sum \xi_{j}^{2}$, which is already in action angle variables and has the remarkable property that the frequency map is very simple.

Here, in order to keep at a minimum level the technicalities, we work without using explicitly action angle variables and we make all the developments in the original Cartesian coordinates $(x, \xi)$. A priori, the main difficulty in doing this consists in solving the homological equation $(3.2)$ : to this end we use here a method developed in [Bam96] which consists in making Fourier developments based on the Hamiltonian flow of the actions. This requires a careful study of the properties of the flow of the actions variables and of the behavior of symbols under such a flow: it will be done in Subsects. 5.2 and 5.3 .

As anticipated in the Introduction, the other difficulties are mainly related to the study of the structure of the actions and of the frequency map.

\section{Properties of $h_{0}$}

In this section we study the properties of the actions variables of $h_{0}$. In this study, a relevant role is played by quasi-homogeneous functions.

Definition 4.1. A function $f \in C^{\infty}\left(\mathbb{R}^{4} \backslash\{0\}\right)$ is said to be quasi-homogeneous of degree $m$ if for any $(x, \xi) \in \mathbb{R}^{4} \backslash\{0\}$ one has

$$
f\left(\lambda x, \lambda^{\ell} \xi\right)=\lambda^{m} f(x, \xi), \quad \forall \lambda>0 .
$$

In the following we will also use functions (of the actions) which are homogeneous in the standard sense.

We remark that if $f$ is quasi-homogeneous of degree $m$ then $\partial_{x}^{\alpha} \partial_{\xi}^{\beta} f$ is quasi-homogeneous of degree $m-|\alpha|-|\beta| \ell$.

Remark 4.2. If $f$ is quasi-homogeneous of some degree $m$ and smooth over the whole $\mathbb{R}^{4}$, then it is also a symbol of class $S_{H R}^{\frac{m}{\ell+1}}$.

The main limitation of the above remark is that a quasi-homogeneous function is $C^{\infty}$ until the origin only if it is a polynomial. Nevertheless, since

\footnotetext{
${ }^{1}$ Of course there are many technical details to verify, but this will be done in the forthcoming sections
} 
we are interested in the behavior at infinity of the symbols, this is not a problem. To make this precise, we fix once for all a cutoff function $\chi$ which is even and $C^{\infty}(\mathbb{R}, \mathbb{R})$, and is supported in $[-1,1]$ and equal to 1 in $\left[-\frac{1}{2}, \frac{1}{2}\right]$.

Definition 4.3. Given a function $f: \mathbb{R}^{4} \rightarrow \mathbb{R}$ we define

$$
f_{\chi}(x, \xi):=(1-\chi(\|a(x, \xi)\|)) f(x, \xi) \text {. }
$$

Remark 4.4. If $f$ is quasi-homogeneous of degree $m$ then $f_{\chi} \in S_{H R}^{\frac{m}{\ell+1}}$.

With a slight abuse of notation, in this Section 4 we will say that $f \in S_{H R}^{m}$ if $f_{\chi} \in S_{H R}^{m}$. Analogously, in Section 5 we will define a new class of symbols, $S_{A N, \delta}^{m}$, and we will say that $f \in S_{A N, \delta}^{m}$ if $f_{\chi} \in S_{A N, \delta}^{m}$.

During the rest of the paper however we will work carefully because the functions to be quantized are everywhere defined, so we have to take into account their behavior on the whole of $\mathbb{R}^{4}$. So starting from Section 6 we will come back to the correct terminology.

We start now the study of the action variables for $h_{0}$. Their properties can be deduced from the general theory of integrable systems (we follow here the ideas of [BF16, CdV80]). Here we give a direct proof of all the properties working on the Hamiltonian $h_{0}$. We recall that the actions, by their definition have the property that the flow they generate is periodic in time with period $2 \pi$.

The action variables can be defined to be the angular momentum

$$
a_{2}(x, \xi):=x_{1} \xi_{2}-x_{2} \xi_{1}
$$

and the radial action, namely the action of the effective Hamiltonian

$$
h_{0}^{*}\left(r, p_{r}, L\right):=\frac{p_{r}^{2}}{2}+V_{L}^{*}(r), \quad V_{L}^{*}(r):=\frac{L^{2}}{2 r^{2}}+\frac{r^{2 \ell}}{2 \ell}
$$

where $r=\|x\|$ and $p_{r}$ is the conjugated momentum. By $L$ we mean here the value taken by the angular momentum, namely we mean that we are on a level surface $a_{2}(x, \xi)=L$.

In order to define $a_{1}$, for $L \neq 0$ we preliminary define

$$
a_{r}=a_{r}(E, L):=\frac{\sqrt{2}}{\pi} \int_{r_{m}}^{r_{M}} \sqrt{E-V_{L}^{*}(r)} d r
$$

where $0<r_{m}<r_{M}$ are the two solutions of the equation

$$
E-V_{L}^{*}(r)=0
$$


Lemma 4.5. For $L>0$, consider the function

$$
a_{1}(E, L):=a_{r}(E, L), \quad L>0 \text {. }
$$

Such function has the following properties:

(1) it extends to a complex analytic function (still denoted by $a_{1}$ ) of $L$ and $E$ in the region

$$
|L|<\left(\frac{2 \ell}{\ell+1} E\right)^{\frac{\ell+1}{2 \ell}}, \quad E>0
$$

(2) for $L<0$ one has

$$
a_{r}(E, L)=a_{1}(E, L)+L
$$

(3) the function $a_{1}(x, \xi):=a_{1}\left(h_{0}(x, \xi), a_{2}(x, \xi)\right)$ is quasi-homogeneous of degree $\ell+1$, so in particular it is of class $C^{1}\left(\mathbb{R}^{4}\right)$;

(4) the map $E \mapsto a_{1}\left(E, a_{2}\right)$ admits an inverse $E=h_{0}\left(a_{1}, a_{2}\right)$ which is analytic in the interior of $\Pi$. Furthermore it is homogeneous of degree $\frac{2 \ell}{\ell+1}$, namely one has

$$
h_{0}\left(\lambda a_{1}, \lambda a_{2}\right)=\lambda^{\frac{2 \ell}{\ell+1}} h_{0}\left(a_{1}, a_{2}\right), \quad \forall \lambda>0 .
$$

The proof of this Lemma is postponed to Appendix A.

Corollary 4.6. Denote $\omega:=\left(\omega_{1}, \omega_{2}\right)$, with $\omega_{i}(a):=\frac{\partial h_{0}}{\partial a_{i}}(a)$, then $\omega_{i}$ are homogeneous of degree

$$
M:=\frac{\ell-1}{\ell+1}
$$

as functions of a. Furthermore $\omega_{i}(x, \xi):=\omega_{i}\left(a_{1}(x, \xi), a_{2}(x, \xi)\right)$ is quasihomogeneous of degree $\ell-1$.

Remark 4.7. One has that $a_{i}$ are quasi-homogeneous functions of order $\ell+1$, and furthermore $a=0$ implies $x=\xi=0$. It follows that

$$
\langle a(x, \xi)\rangle \simeq \mathrm{k}_{0}(x, \xi) .
$$




\section{Symbols, Fourier expansions and Pseudo- differential calculus}

\subsection{A class of symbols}

In the following we will need a class of symbols slightly more general than those of Definition 2.2.

First of all we fix positive parameters $\epsilon, \delta$ fulfilling

$$
\frac{\ell-2}{\ell+1}<\delta<\frac{\ell-1}{\ell+1}=M, \quad 0<\epsilon<M-\delta
$$

further requirements on $\delta$ and $\epsilon$ will be specified later on (see equation 6.2 and Remark (8.12) below). We also define

$$
\delta_{1}:=\delta-\frac{\ell-2}{\ell+1}, \quad \delta_{2}:=\delta_{1}+\frac{\ell-1}{\ell+1},
$$

and remark that

$$
\delta_{1}+\delta_{2}=2 \delta-\frac{\ell-3}{\ell+1} \Longrightarrow \frac{\ell-1}{\ell+1}<\delta_{1}+\delta_{2}<1 .
$$

Definition 5.1. Given $f \in C^{\infty}\left(\mathbb{R}^{4}\right)$, we will write $f \in S_{A N, \delta}^{m}$ if $\forall \alpha, \beta \in \mathbb{N}^{2}$, there exists $C_{\alpha, \beta}>0$ s.t.

$$
\left|\partial_{x}^{\alpha} \partial_{\xi}^{\beta} f(x, \xi)\right| \leq C_{\alpha, \beta}\left(\mathrm{k}_{0}(x, \xi)\right)^{m-\delta_{1}|\alpha|-\delta_{2}|\beta|},
$$

with $\delta_{1}, \delta_{2}$ given by (5.2). We will say that an operator $F$ is a pseudodifferential operator of class $O P S_{A N, \delta}^{m}$ if there exists symbol $f \in S_{A N, \delta}^{m}$ s.t. $F=O p^{w}(f)$.

The smallest constants $C_{\alpha, \beta}$ s.t. Eq. (5.4) holds form a family of seminorms for the symbols of this class. However in order to get the standard algebra properties it is more convenient to use a different definition.

Definition 5.2. Let $f \in S_{A N, \delta}^{m}$, then, $\forall \alpha, \beta \in \mathbb{N}^{d}$, we put

$$
\|f\|_{\alpha, \beta}^{(m)}:=\sup _{x, \xi} \sup _{\left|\alpha^{\prime}\right| \leq|\alpha|,\left|\beta^{\prime}\right| \leq|\beta|}\left|\partial_{x}^{\alpha^{\prime}} \partial_{\xi}^{\beta^{\prime}} f(x, \xi)\right|\left(\mathrm{k}_{0}(x, \xi)\right)^{-\left(m-\left|\alpha^{\prime}\right| \delta_{1}-\left|\beta^{\prime}\right| \delta_{2}\right)} .
$$

Remark 5.3. With this definition we have

$$
\|f g\|_{\alpha, \beta}^{\left(m+m^{\prime}\right)} \leq\|f\|_{\alpha, \beta}^{(m)}\|g\|_{\alpha, \beta}^{\left(m^{\prime}\right)}
$$

for any couple of symbols $f \in S_{A N, \delta}^{m}, g \in S_{A N, \delta}^{m^{\prime}}$. 
Lemma 5.4. Let $m, m^{\prime} \in \mathbb{R}, F=O p^{w}(f) \in O P S_{A N, \delta}^{m}, G=O p^{w}(g) \in$ $O P S_{A N, \delta}^{m^{\prime}}$. Then $F G \in O P S_{A N, \delta}^{m+m^{\prime}}$. Denote by $f \sharp g$ its symbol, then it admits the asymptotic expansion

$f \sharp g \sim \sum_{j \geq 0}(f \sharp g)_{j}$,
$(f \sharp g)_{j}:=\frac{1}{\mathrm{i}^{j}} \sum_{|\alpha|+|\beta|=j}\left(\frac{1}{2}\right)^{|\alpha|}\left(-\frac{1}{2}\right)^{|\beta|}\left(\partial_{x}^{\beta} \partial_{\xi}^{\alpha} f\right)\left(\partial_{x}^{\alpha} \partial_{\xi}^{\beta} g\right) \in S_{A N, \delta}^{m+m^{\prime}-\left(\delta_{1}+\delta_{2}\right) j}, \quad j \geq 0$.

Furthermore, $\forall n, \alpha, \beta, \exists \alpha^{\prime}, \beta^{\prime}$ and $C>0$ s.t.

$$
\left\|f \sharp g-\sum_{j=1}^{n-1}(f \sharp g)_{j}\right\|_{\alpha, \beta}^{\left(m-m^{\prime}-n\left(\delta_{1}+\delta_{2}\right)\right)} \leq C\|f\|_{\alpha^{\prime}, \beta^{\prime}}^{m}\|g\|_{\alpha^{\prime}, \beta^{\prime}}^{m^{\prime}} .
$$

Lemma 5.5. (Calderon Vaillancourt theorem) Let $f \in S_{A N, \delta}^{m}$, then $\forall s \in \mathbb{R}$, $O p^{w}(f) \in B\left(\mathcal{H}^{s+m} ; \mathcal{H}^{s}\right)$ and there exist $C, \alpha, \beta$, s.t.

$$
\left\|O p^{w}(f)\right\|_{s+m, s} \leq C\|f\|_{\alpha, \beta}^{(m)}
$$

\subsection{Flow of the actions}

In order to develop perturbation theory, we will make use of a Fourier development based on the flow of the actions (following the ideas of [Bam96]). For this reason such a flow has to be studied quite in detail.

Let $a_{i}$ be the $i$-th action. Consider the corresponding Hamilton equations, namely

$$
\dot{\xi}=-\frac{\partial a_{i}}{\partial x}, \quad \dot{x}=\frac{\partial a_{i}}{\partial \xi}
$$

and denote by $\phi_{a_{i}}^{\varphi}$ the corresponding time $\varphi$ flow.

We are now going to study $\phi_{a_{i}}^{\varphi}$ and, for $f \in S_{A N, \delta}^{m}, f \circ \phi_{a_{i}}^{\varphi}$. We concentrate on the non trivial action $a_{1}$, but all what follows is only based on the property that $a_{1}$ is quasi-homogeneous of degree $\ell+1$, so it trivially holds also for $a_{2}$. We will also distinguish between the $x$ and the $\xi$ components of the flow, so we will write

$$
\phi_{a_{1}}^{\varphi}(x, \xi)=\left(X^{\varphi}(x, \xi), \Xi^{\varphi}(x, \xi)\right) .
$$

Lemma 5.6. $\forall \varphi \in \mathbb{R}$ the function $X^{\varphi}$ is quasi-homogeneous of degree 1 and $\Xi^{\varphi}$ is quasi-homogeneous of degree $\ell$. 
Proof. For $\lambda>0$, consider

$$
X_{\lambda}^{\varphi}(x, \xi):=\frac{1}{\lambda} X^{\varphi}\left(\lambda x, \lambda^{\ell} \xi\right), \quad \Xi_{\lambda}^{\varphi}(x, \xi):=\frac{1}{\lambda^{\ell}} X^{\varphi}\left(\lambda x, \lambda^{\ell} \xi\right) .
$$

One has

$$
\left.X_{\lambda}^{\varphi}(x, \xi)\right|_{\varphi=0}=x,\left.\quad \Xi_{\lambda}^{\varphi}(x, \xi)\right|_{\varphi=0}=\xi .
$$

Furthermore, since $\frac{\partial a_{1}}{\partial \xi}$ is quasi homogeneous of degree 1, one gets

$$
\begin{array}{r}
\quad \frac{d X_{\lambda}^{\varphi}}{d \varphi}(x, \xi)=\frac{1}{\lambda} \frac{d X^{\varphi}}{d \varphi}\left(\lambda x, \lambda^{\ell} \xi\right)=\frac{1}{\lambda} \frac{\partial a_{1}}{\partial \xi}\left(X^{\varphi}\left(\lambda x, \lambda^{\ell} \xi\right), \Xi^{\varphi}\left(\lambda x, \lambda^{\ell} \xi\right)\right) \\
=\frac{\partial a_{1}}{\partial \xi}\left(\frac{1}{\lambda} X^{\varphi}\left(\lambda x, \lambda^{\ell} \xi\right), \frac{1}{\lambda^{\ell}} \Xi^{\varphi}\left(\lambda x, \lambda^{\ell} \xi\right)\right)=\frac{\partial a_{1}}{\partial \xi}\left(X_{\lambda}^{\varphi}(x, \xi), \Xi_{\lambda}^{\varphi}(x, \xi)\right),
\end{array}
$$

which shows that $\left(X^{\varphi}, \Xi^{\varphi}\right)$ and $\left(X_{\lambda}^{\varphi}, \Xi_{\lambda}^{\varphi}\right)$ satisfy the same Cauchy problem, so they coincide.

It follows that if $f$ is quasi-homogeneous of some degree, then $f \circ \phi_{a_{1}}^{\varphi}$ is quasi-homogeneous of the same degree. It is also easy to see that if $f \in S_{H R}^{m}$ then the same is true for $f \circ \phi_{a_{1}}^{\varphi}$ (more precisely, according to our abuse of notation, for $\left.\left(f \circ \phi_{a_{1}}^{\varphi}\right)_{\chi}\right)$. We are now going to show that also the class $S_{A N, \delta}^{m}$ is left invariant by the composition with $\phi$ (up to a cutoff close to the origin).

Lemma 5.7. If $f \in S_{A N, \delta}^{m}$ then $f \circ \phi_{a_{1}}^{\varphi} \in S_{A N, \delta}^{m}$.

Proof. We are going to study $\frac{\partial^{|\alpha|}}{\partial x^{\alpha}} f \circ \phi_{a_{1}}^{\varphi}$; the other derivatives can be studied in the same way. We are going to prove that

$$
\frac{\partial^{|\alpha|}}{\partial x^{\alpha}} f \circ \phi_{a_{1}}^{\varphi}=\sum_{\beta, \gamma} \Phi_{\gamma \beta} \frac{\partial^{|\gamma+\beta|} f}{\partial x^{\gamma} \partial \xi^{\beta}} \circ \phi_{a_{1}}^{\varphi}
$$

with $\Phi_{\gamma \beta} \in S_{H R}^{|\beta| \delta_{2}+(|\gamma|-|\alpha|) \delta_{1}}$, and $|\gamma|+|\beta| \leq|\alpha|$. From this structure one immediately has

$$
\left|\frac{\partial^{|\alpha|}}{\partial x^{\alpha}} f \circ \phi_{a_{1}}^{\varphi}\right| \lesssim \mathrm{k}_{0}^{m-|\alpha| \delta_{1}}
$$

and, adding the estimates of the other derivative, the thesis. We now prove (5.13). To shorten the notation denote $f_{\gamma \beta}:=\frac{\partial^{|\gamma+\beta|} f}{\partial x^{\gamma} \partial \xi^{\beta}}$ and compute (to be determined)

$$
\begin{aligned}
\frac{\partial}{\partial x_{1}}\left(f_{\gamma \beta} \circ \phi_{a_{1}}^{\varphi} \Phi_{\gamma \beta}\right) & =\left(\frac{\partial X^{\varphi}}{\partial x_{1}} \cdot \frac{\partial f_{\gamma \beta}}{\partial x} \circ \phi_{a_{1}}^{\varphi}+\frac{\partial \Xi^{\varphi}}{\partial x_{1}} \cdot \frac{\partial f_{\gamma \beta}}{\partial \xi} \circ \phi_{a_{1}}^{\varphi}\right) \Phi_{\gamma \beta} \\
& +f_{\gamma \beta} \circ \phi_{a_{1}}^{\varphi} \frac{\partial \Phi_{\gamma \beta}}{\partial x_{1}}
\end{aligned}
$$


The second line has the wanted structure. We compute the structure of the first line. Consider its first term (namely $\frac{\partial X^{\varphi}}{\partial x_{1}} \cdot \frac{\partial f_{\gamma \beta}}{\partial x} \circ \phi_{a_{1}}^{\varphi}$ ) which is actually the sum of two terms, corresponding to the different components of the gradient and of $X$. The first of these two terms has the wanted structure with

$$
\Phi_{(\gamma+(1,0)) \beta}:=\frac{\partial\left(X^{\varphi}\right)_{1}}{\partial x_{1}} \Phi_{\gamma \beta} \in S_{H R}^{|\beta| \delta_{2}+(|\gamma|-|\alpha|) \delta_{1}}
$$

(since $\frac{\partial\left(X^{\varphi}\right)_{1}}{\partial x_{1}}$ is homogeneous of degree zero), which has correct order since $|\gamma+(1,0)|=|\gamma|+1$ and the new $\alpha$ has modulus $|\alpha|+1$. Consider now the first of the terms containing $\Xi$. It has the wanted structure with

$$
\Phi_{\gamma(\beta+(1,0))}:=\frac{\partial\left(\Xi^{\varphi}\right)_{1}}{\partial x_{1}} \Phi_{\gamma \beta} \in S_{H R}^{|\beta| \delta_{2}+(|\gamma|-|\alpha|) \delta_{1}+\frac{\ell}{\ell+1}-\frac{1}{\ell+1}}
$$

(since $\Xi^{\varphi}$ is homogeneous of degree $\ell$ ). So the order is

$$
|\beta| \delta_{2}+(|\gamma|-(|\alpha|+1)) \delta_{1}+\delta_{1}+\frac{\ell-1}{\ell+1}=(|\beta|+1) \delta_{2}+(|\gamma|-(|\alpha|+1)) \delta_{1}
$$

as it should be.

From now on we will need to consider the composition of the flows of $a_{1}$ and $a_{2}$. So we denote

$$
\varphi:=\left(\varphi_{1}, \varphi_{2}\right), \quad \phi_{a}^{\varphi}:=\phi_{a_{1}}^{\varphi_{1}} \circ \phi_{a_{2}}^{\varphi_{2}} .
$$

and remark that $\phi_{a}^{\varphi}$ is $2 \pi$ periodic in each one of the $\varphi$ 's.

Remark 5.8. The result of Lemma 5.7 holds also for the joint flow $\phi_{a}^{\varphi}$.

\subsection{Fourier expansion and summable symbols}

Following [Bam96] we consider the Fourier development defined by the flow $\phi_{a}^{\varphi}$.

Definition 5.9. Let $f \in S_{A N, \delta}^{m}$, then, for $k \in \mathbb{Z}^{2}$,

$$
\hat{f}_{k}(x, \xi):=\frac{1}{4 \pi^{2}} \int_{\mathbb{T}^{2}} f\left(\phi_{a}^{\varphi}(x, \xi)\right) e^{-\mathrm{i} k \cdot \varphi} d \varphi
$$

is called the $k$-th Fourier coefficient of $f$.

Remark 5.10. From standard theory of Fourier expansions one has, for fixed $(x, \xi) \in \mathbb{R}^{4}$,

$$
f\left(\phi_{a}^{\varphi}(x, \xi)\right)=\sum_{k \in \mathbb{Z}^{2}} \hat{f}_{k}(x, \xi) e^{\mathrm{i} k \cdot \varphi}
$$

so that, in particular

$$
f(x, \xi)=\sum_{k \in \mathbb{Z}^{2}} \hat{f}_{k}(x, \xi)
$$


Remark 5.11. Let $g$ be a function invariant under the flow of the actions, then, for any function $f$, one has that the $k$-th Fourier coefficient of $g f$, namely $\widehat{(g f)}{ }_{k}$, is $g \hat{f}_{k}$.

The 0 -th Fourier coefficient of a symbol $f \in \mathcal{S F}_{N}^{m}$ is essentially a function of the actions only. To give a precise statement, we proceed as in [Cha86]; introduce an open cone $\mathcal{V}$, s.t. $\overline{\mathcal{V}} \backslash\{0\}$ is contained in the interior of $\Pi$ and $\overline{\mathcal{C}} \backslash\{0\}$ is contained in the interior of $\mathcal{V}$, then we have the following Lemma.

Lemma 5.12. Let $f \in S_{A N, \delta}^{m}$, with the property that $f \circ \phi_{a}^{\varphi}=f, \forall \varphi \in \mathbb{T}^{2}$, then there exists a symbol $f_{c} \in S_{C}^{m, \varsigma}$, with $\varsigma=1-(M-\delta)$, s.t.

$$
f(x, \xi)=f_{c}(a(x, \xi)), \quad \forall(x, \xi): a(x, \xi) \in \mathcal{V} \cap B_{1}^{c},
$$

where, given $r>0, B_{r}^{c}$ is the complement of the ball of radius $r$ in $\mathbb{R}^{2}$.

The proof of this Lemma is postponed to Appendix B.

From now on, if $f: \mathbb{R}^{4} \rightarrow \mathbb{R}$ is invariant with respect to the flow of the actions, we will simply say that it is a function of the actions only, and we will denote by $f_{c} \in S_{C}^{m, \varsigma}$ the symbol such that (5.17) holds.

Definition 5.13. For $m \in \mathbb{R}$ and $N \in \mathbb{N}$, the set of the symbols $f \in S_{A N, \delta}^{m}$ s.t. $\forall \alpha, \beta \in \mathbb{N}^{2}$,

$$
\|f\|_{\alpha, \beta, N}^{(m)}:=\sum_{k \in \mathbb{Z}^{2}}\langle k\rangle^{N}\left\|\hat{f}_{k}\right\|_{\alpha, \beta}^{(m)}<\infty
$$

will be denoted by $\mathcal{S F}_{N}^{m}$. The seminorms $\|\cdot\|_{\alpha, \beta}^{(m)}$ are defined by (5.5).

We will say that an operator $F$ is a pseudo-differential operator of class $\mathcal{O P S F}_{N}^{m}$ if there exists symbol $f \in \mathcal{S F}_{N}^{m}$ s.t. $F=O p^{w}(f)$. $\mathcal{S} \mathcal{F}_{N}^{m}$ :

The following result relates the symbols of class $S_{H R}^{m}$ with those of class

Lemma 5.14. Let $f \in S_{H R}^{m}$, then for any $\alpha, \beta \in \mathbb{N}^{2}$ and $N \in \mathbb{N}$ there exists $C>0$, independent of $f$, such that

$$
\sup _{x, \xi} \sup _{\left|\alpha^{\prime}\right| \leq|\alpha|,\left|\beta^{\prime}\right| \leq|\beta|}\left|\partial_{x}^{\alpha} \partial_{\xi}^{\beta} \hat{f}_{k}(x, \xi)\right|\left(\mathrm{k}_{0}(x, \xi)\right)^{-\left(m-\frac{|\alpha|}{\ell+1}-\frac{|\beta| \ell}{\ell+1}\right)} \leq C\langle k\rangle^{-N} .
$$

In particular, $f \in \mathcal{S F}_{N}^{m}$ for all $N$.

Proof. In order to prove that 5.19 holds, we proceed as usual, by integrating by parts in (5.15). To fix ideas consider the case $k_{1}>0, k_{2}>0$. Denote just 
for this proof, $\partial:=\partial_{\varphi_{1}}+\partial_{\varphi_{2}}$, then exploiting $\partial e^{-\mathrm{i} k \cdot \varphi}=-\mathrm{i}|k| e^{-\mathrm{i} k \cdot \varphi}$, we have $\forall N \geq 1$

$$
\begin{aligned}
\hat{f}_{k} & =\frac{1}{4 \pi^{2}} \int_{\mathbb{T}^{2}}\left(f \circ \phi_{a}^{\varphi}\right) \frac{1}{(-\mathrm{i}|k|)^{N}} \partial^{N} e^{\mathrm{i} k \cdot \varphi} d \varphi \\
& =\frac{1}{(\mathrm{i}|k|)^{N}} \frac{1}{4 \pi^{2}} \int_{\mathbb{T}^{2}} \partial^{N}\left(f \circ \phi_{a}^{\varphi}\right) e^{\mathrm{i} k \cdot \varphi} d \varphi
\end{aligned}
$$

In order to estimate $\partial^{N}\left(f \circ \phi_{a}^{\varphi}\right)$, we start by considering

$$
\partial_{\varphi_{i}}\left(f \circ \phi_{a}^{\varphi}\right)=\left\{f ; a_{i}\right\} \circ \phi_{a}^{\varphi}=\left(a d_{a_{i}} f\right) \circ \phi_{a}^{\varphi},
$$

where

$$
a d_{a_{i}} f:=\left\{f ; a_{i}\right\}
$$

From this one has

$$
\partial^{N}\left(f \circ \phi_{a}^{\varphi}\right)=\left[\left(a d_{a_{1}}+a d_{a_{2}}\right)^{N} f\right] \circ \phi_{a}^{\varphi} .
$$

Now, if $f \in S_{H R}^{m}$ one has

$$
a d_{a_{i}} f=\frac{\partial a_{i}}{\partial \xi} \cdot \frac{\partial f}{\partial x}-\frac{\partial a_{i}}{\partial x} \cdot \frac{\partial f}{\partial \xi} \in S_{H R}^{m}
$$

due to Lemma 5.17, thus $\partial^{N}\left(f \circ \phi_{a}^{\varphi}\right) \in S_{H R}^{m}$ for any $N$. By Lemma 5.7, this implies

$$
\partial^{N}\left(f \circ \phi_{a}^{\varphi}\right) \in S_{H R}^{m},
$$

from which (5.19) immediately follows.

Remark 5.15. We point out that $f \in S_{A N, \delta}^{m}$ does not imply $f \in \mathcal{S F}_{N}^{m}$; in particular, arguing as in the proof of Lemma 5.14, from $f \in S_{A N, \delta}^{m}$ one can only deduce that

$$
\|f\|_{\alpha, \beta, N}^{(m+(M-\delta) N)}<+\infty \quad \forall \alpha, \beta \in \mathbb{N}^{2}, \quad \forall N \in \mathbb{N} .
$$

This is the main reason why we work in the stronger class $\mathcal{S F}_{N}^{m}$.

Lemma 5.16. Let $f \in \mathcal{S F}_{N}^{m}$ and $g \in \mathcal{S F}_{N}^{m^{\prime}}$. Then $f g \in \mathcal{S F}_{N}^{m+m^{\prime}}$, and $\forall N \in \mathbb{N}$ there exists $C_{N}>0$ such that $\forall \alpha, \beta \in \mathbb{N}^{2}$

$$
\|f g\|_{\alpha, \beta, N}^{\left(m+m^{\prime}\right)} \leq C_{N}\|f\|_{\alpha, \beta, N}^{(m)}\|g\|_{\alpha, \beta, N}^{\left(m^{\prime}\right)} .
$$


Proof. First of all we observe that,

$$
(f g) \circ \phi_{a}^{\varphi}=\left(f \circ \phi_{a}^{\varphi}\right)\left(g \circ \phi_{a}^{\varphi}\right)=\sum_{k, k^{\prime} \in \mathbb{Z}^{2}} \hat{f}_{k} \hat{g}_{k^{\prime}} e^{\mathrm{i}\left(k+k^{\prime}\right) \cdot \varphi},
$$

thus we have

$$
\begin{array}{r}
\|f g\|_{0,0, N}^{\left(m+m^{\prime}\right)} \leq \sum_{k, k^{\prime}}\left\langle k+k^{\prime}\right\rangle^{N}\left|\hat{f}_{k}\right|\left|\hat{g}_{k}\right| \mathrm{k}_{0}^{-\left(m+m^{\prime}\right)} \\
\leq C_{N} \sum_{k, k^{\prime}}\langle k\rangle^{N}\left\langle k^{\prime}\right\rangle^{N}\left|\hat{f}_{k}\right|\left|\hat{g}_{k}\right| \mathrm{k}_{0}^{-\left(m+m^{\prime}\right)} \leq C_{N}\|f\|_{0,0, N}^{(m)}\|g\|_{0,0, N}^{\left(m^{\prime}\right)} .
\end{array}
$$

Working in the same way with the derivatives and exploiting Remark 5.3 , one gets the result.

The following results can be proved by simple variations on the standard arguments

Lemma 5.17. Let $m, m^{\prime} \in \mathbb{R}, F=O p^{w}(f) \in \mathcal{O P S F}_{N}^{m}, G=O p^{w}(g) \in$ $\mathcal{O P S F}_{N}^{m^{\prime}}$. Then $F G \in \mathcal{O P S F}_{N}^{m+m^{\prime}}$. Denote by $f \sharp g$ its symbol, then it admits the same asymptotic expansion as in (5.6), but now

$$
(f \sharp g)_{j} \in \mathcal{S F}_{N}^{m+m^{\prime}-\left(\delta_{1}+\delta_{2}\right) j} \quad \forall j \in \mathbb{N} .
$$

Corollary 5.18. One has

$$
\{f ; g\}_{M}=\{f ; g\}+\mathcal{S} \mathcal{F}_{N}^{m+m^{\prime}-3\left(\delta_{1}+\delta_{2}\right)} .
$$

Definition 5.19. In what follows we will denote

$$
a d_{g}^{M} a:=\{a ; g\}_{M}
$$

which is well defined in anyone of the classes of symbols we are using.

Given a self-adjoint pseudo-differential operator $G \in \mathcal{O} \mathcal{P} \mathcal{S} \mathcal{F}_{N}^{\eta}$, we consider the unitary group generated by $-\mathrm{i} G$, which is denoted, as usual, by $\mathrm{e}^{-\mathrm{i} \tau G}, \tau \in \mathbb{R}$.

The following version of Egorov Theorem holds:

Lemma 5.20. Fix $\eta \in \mathbb{R}$, and let $g \in \mathcal{S F}_{N}^{\eta}$ be a real valued symbol, denote $G=O p^{w}(g)$, then $\forall \tau \in[-1,1]$

$$
\text { (1) If } \eta \leq \delta+\frac{2}{\ell+1} \text {, then } e^{\mathrm{i} \tau G} \in \mathcal{B}\left(\mathcal{H}^{s} ; \mathcal{H}^{s}\right) \quad \forall s \geq 0
$$


(2) Assume $\eta<\delta_{1}+\delta_{2}$, and let $f \in \mathcal{S F}_{N}^{m}, F=O p^{w}(f)$, then

$$
e^{\mathrm{i} \tau G} F e^{-\mathrm{i} \tau G}=: F^{\prime} \in \mathcal{O} \mathcal{P} \mathcal{S} \mathcal{F}_{N}^{m} .
$$

Furthermore, denoting by $f^{\prime}$ its symbol, for any $n \in \mathbb{N}$ one has

$$
f^{\prime}=\sum_{0 \leq j \leq n} \frac{\tau^{j}\left(a d_{g}^{M}\right)^{j} f}{j !}+\mathcal{S} \mathcal{F}_{N}^{m+(n+1)\left(\eta-\left(\delta_{1}+\delta_{2}\right)\right)} .
$$

In particular, one has

$$
f^{\prime}=f+\{f ; g\}_{M}+\mathcal{S} \mathcal{F}_{N}^{m+2\left(\eta-\delta_{1}-\delta_{2}\right)} .
$$

Definition 5.21. As a general notation, given two symbols $f \in \mathcal{S F}_{N}^{m}$ and $g \in \mathcal{S F}_{N}^{\eta}$ with $\eta<\delta_{1}+\delta_{2}$, we will denote by $f^{\prime} \in \mathcal{S F}_{N}^{m}$ (namely with a prime) the symbol such that

$$
e^{\mathrm{i} G} O p^{w}(f) e^{-\mathrm{i} G}=O p^{w}\left(f^{\prime}\right) .
$$

\section{The normal form lemma}

From now on we abandon our abuse of notation related to the cutoff at the origin and a symbol will always be a $C^{\infty}$ function fulfilling the required estimates everywhere.

We give the following definition:

Definition 6.1. We say that a symbol $z \in \mathcal{S F}_{N}^{m}$ is in resonant normal form if $\forall k \in \mathbb{Z}^{2} \backslash\{0\}$ its Fourier coefficients satisfy the following:

$$
\text { supp } \hat{z}_{k} \subseteq \mathcal{R}_{k}:=\left\{(x, \xi)|| \omega(a) \cdot k \mid \leq\|a\|^{\delta}\|k\|, \quad\|k\| \leq\|a\|^{\epsilon}\right\},
$$

where supp denotes the support of the function in argument.

This section is devoted to the proof of the following result:

Lemma 6.2 (Normal form). Let $m=\frac{2 \ell}{\ell+1}-\mathfrak{e}, \mathfrak{e}>0$. Furthermore, suppose that $\epsilon, \delta$ satisfy (5.1) and that

$$
M-\delta<\min \left\{\frac{\mathfrak{e}}{3}, \frac{2}{7}\right\},
$$

and define, as in Eq. 2.18,

$$
\rho=\min \{\mathfrak{e}-3(M-\delta), 2-7(M-\delta)\} .
$$


Fix $\mathrm{N}$ arbitrarily large, take $N$ s.t. $N \epsilon \geq \mathrm{N} \rho$, then there exists a sequence of self-adjoint pseudo-differential operators $\left\{G_{n}\right\}_{n=1}^{\mathrm{N}-1}, G_{n} \in \mathcal{O} \mathcal{P} \mathcal{S} \mathcal{F}_{N}^{m-n \rho-\delta}$, such that the operator

$$
\mathcal{U}_{n}:=e^{\mathrm{i} G_{1}} \circ \cdots \circ e^{\mathrm{i} G_{n}}
$$

conjugates $H$ to $H_{n}=O p^{w}\left(h_{n}+w_{n}\right)$, where $w_{n} \in \mathcal{S} \mathcal{F}_{0}^{m-N \rho}$ is a real symbol and $h_{n}=h_{0}+z^{(n)}+v_{n}$ has the following properties:

1. $v_{n} \in \mathcal{S F}_{N}^{m-n \rho}$ is a real symbol

2. $z^{(n)}=\left\langle z^{(n)}\right\rangle+z_{n}^{(r e s)}$ is in resonant normal form, and there exists a sequence $\left\{z_{j}\right\}_{j \in \mathbb{N}}$ of real valued smooth functions $z_{j} \in \mathcal{S F}_{N}^{m-(j-1) \rho}$, which are functions of the actions only, such that

$$
\left\langle z^{(n)}\right\rangle=\sum_{j=1}^{n} z_{j} .
$$

The proof of Lemma 6.2 is obtained following the same approach of [BLM20b, PS10], which we are now going to adapt.

Consider again the cutoff function $\chi$ fixed above. With its help we define, for $k \in \mathbb{Z}^{2} \backslash\{0\}$

$$
\begin{gathered}
\chi_{k}(a):=\chi\left(\frac{\omega(a) \cdot k}{\|a\|^{\delta}\|k\|}\right), \\
d_{k}(a):=\frac{1}{\mathrm{i} \omega(a) \cdot k}\left(1-\chi_{k}(a)\right), \\
\tilde{\chi}_{k}(a):=\chi\left(\frac{\|k\|}{\|a\|^{\epsilon}}\right) .
\end{gathered}
$$

Of course they will be considered as functions of $(x, \xi)$ (by the substitution $a=a(x, \xi))$. Furthermore, given $f \in \mathcal{S F}_{N}^{m}$, we define

$$
\begin{aligned}
\langle f\rangle & :=(1-\chi(\|a\|)) \hat{f}_{0}, \\
f^{(r e s)} & :=\sum_{k \in Z^{2} \backslash\{0\}}(1-\chi(\|a\|)) \chi_{k} \tilde{\chi}_{k} \hat{f}_{k}, \\
f^{(n r)}: & =\sum_{k \in Z^{2}}(1-\chi(\|a\|))\left(1-\chi_{k}\right) \tilde{\chi}_{k} \hat{f}_{k}, \\
f^{(S)} & :=\sum_{k \in Z^{2} \backslash\{0\}}(1-\chi(\|a\|))\left(1-\tilde{\chi}_{k}\right) \hat{f}_{k}+\chi(\|a\|) f,
\end{aligned}
$$

so that one has

$$
f=\langle f\rangle+f^{(n r)}+f^{(r e s)}+f^{(S)} .
$$

In order to show that each term is a symbol, we need a few preliminaries. 
Lemma 6.3. One has $(1-\chi(\|a\|)) \chi_{k} \in S_{A N, \delta}^{0}$, furthermore for any $\alpha, \beta$ there exists $C$ s.t. $\forall k \in \mathbb{Z}^{2} \backslash\{0\}$ one has

$$
\left\|(1-\chi(\|a\|)) \chi_{k}\right\|_{\alpha, \beta}^{(0)} \leq C .
$$

Proof. First of all, observe that since $(1-\chi(\|a\|))$ is a symbol of class $S_{H R}^{0}$ it is enough to study $\chi_{k}$ in the support of $(1-\chi(\|a\|))$. Denote

$$
t_{k}(a):=\frac{\omega(a) \cdot k}{\|k\|\|a\|^{\delta}}
$$

which is a homogeneous function of degree $M-\delta>0$; then one has $\chi_{k}=\chi^{\circ} t_{k}$, and by the Faà di Bruno formula

$$
\left|\partial_{x}^{\alpha}\left(\chi \circ t_{k}\right)\right| \simeq \sum_{j=1}^{|\alpha|} \sum_{\gamma_{1}+\ldots+\gamma_{j}=\alpha}\left|\chi^{(j)} \circ t_{k}\right| \prod_{i=i}^{j}\left|\partial_{x}^{\gamma_{i}} t_{k}\right| .
$$

Now, by quasi homogeneity, one has

$$
\left|\partial_{x}^{\gamma_{i}} t_{k}\right| \lesssim\|a\|^{M-\delta-\frac{\left|\gamma_{i}\right|}{\ell+1}} \lesssim\|a\|^{\left|\gamma_{i}\right|\left(M-\delta-\frac{1}{\ell+1}\right)} \lesssim\|a\|^{-\left|\gamma_{i}\right| \delta_{1}}
$$

and therefore

$$
\prod_{i=i}^{j}\left|\partial_{x}^{\gamma_{i}} t_{k}\right| \lesssim\|a\|^{-|\alpha| \delta_{1}}
$$

Remark now, that, in the support of $1-\chi(\|a\|)$, by Remark 4.7 one has $\langle a\rangle \simeq \mathrm{k}_{0}$, thus one has $\prod_{i=i}^{j}\left|\partial_{x}^{\gamma_{i}} t_{k}\right| \lesssim \mathrm{k}_{0}^{-|\alpha| \delta_{1}}$, which in turn implies

$$
\left|\partial_{x}^{\alpha}\left(\chi \circ t_{k}\right)\right| \lesssim \mathrm{k}_{0}^{-|\alpha| \delta_{1}} \quad \forall(x, \xi), \quad \forall k \neq 0 .
$$

Similar estimates hold for the $\xi$ derivatives and for the mixed derivatives, and this implies the thesis.

Lemma 6.4. One has $\tilde{\chi}_{k} \in S_{H R}^{0}$, furthermore all its seminorms are bounded uniformly with respect to $k$.

Proof. We proceed as in the proof of the preceding Lemma, except that we redefine

$$
t_{k}:=\frac{\|k\|}{\|a\| \epsilon} .
$$

Thus one gets again formula (6.8). In this case one has

$$
\left|\partial_{x}^{\gamma_{i}} t_{k}\right|=\|k\|\left|\partial_{x}^{\gamma_{i}} \frac{1}{\|a\|^{\epsilon}}\right| \lesssim\|k\|\|a\|^{-\left(\epsilon+\frac{\left|\gamma_{i}\right|}{\ell+1}\right)}
$$


and thus,

$$
\prod_{i=1}^{j}\left|\partial_{x}^{\gamma_{i}} t_{k}\right| \lesssim\left(\frac{\|k\|}{\|a\|^{\epsilon}}\right)^{j} \frac{1}{\|a\|^{\frac{|\alpha|}{\ell+1}}}
$$

but, on the support of $\tilde{\chi} \mathrm{ot}_{k}$, one has $\frac{\|k\|}{\|a\| \epsilon}<1$, which also implies $\|a\|>(\|k\|)^{1 / \epsilon} \geq 1$, thus we also have, on the whole of $\mathbb{R}^{4}$,

$$
\left|\partial_{x}^{\alpha}\left(\chi \circ t_{k}\right)\right| \lesssim \frac{1}{\mathrm{k}_{0}^{\frac{|\alpha|}{\ell+1}}} .
$$

Similar estimates hold for the $\xi$ derivatives, for the mixed derivatives and this implies the thesis.

Remark 6.5. The results of this Lemma and of Lemma 6.6 below are the main reason for the introduction of the class of symbols of Definition 5.1

Lemma 6.6. One has $(1-\chi(\|a\|)) d_{k} \in S_{A N, \delta}^{-\delta}$, furthermore all its seminorms are bounded uniformly with respect to $k$.

Proof. We study the $x$ derivatives of $d_{k}$, all the others can be estimated exactly in the same way. Arguing as in the proof of Lemma 6.3, again we restrict the study of $d_{k}$ to the support of $(1-\chi(\|a\|))$. From Leibniz formula we have

$$
\left|\partial_{x}^{\alpha} d_{k}\right| \simeq \sum_{\gamma \leq \alpha}\left|\partial_{x}^{\gamma} \frac{1}{\omega \cdot k}\right|\left|\partial_{x}^{\alpha-\gamma}\left(1-\chi_{k}\right)\right| .
$$

Now, one has by Faà di Bruno formula

$$
\left|\partial_{x}^{\gamma} \frac{1}{\omega \cdot k}\right| \simeq \sum_{j=1}^{|\gamma|} \sum_{\nu_{1}+\ldots+\nu_{j}=\gamma} \frac{1}{|\omega \cdot k|^{j+1}} \prod_{i=1}^{j}\left|\partial_{x}^{\nu_{i}} \omega \cdot k\right| .
$$

Now, since we work in the support of $1-\chi_{k}$ which contains also the support of $\partial_{x}^{\alpha-\gamma} \chi_{k}$, we have, in this domain intersected with $\|a\|>\frac{1}{2}$,

$\frac{1}{|\omega \cdot k|^{j+1}} \lesssim\|a\|^{-\delta(j+1)}\|k\|^{-(j+1)} \lesssim \mathrm{k}_{0}^{-\delta(j+1)}\|k\|^{-(j+1)}, \quad\left|\partial_{x}^{\nu_{i}} \omega \cdot k\right| \lesssim \mathrm{k}_{0}^{M-\frac{\left|\nu_{i}\right|}{\ell+1}}\|k\|$,

and therefore

$$
\left|\partial_{x}^{\gamma} \frac{1}{\omega \cdot k}\right| \lesssim \mathrm{k}_{0}^{-\delta+|\gamma|\left(M-\delta-\frac{1}{\ell+1}\right)} \lesssim \mathrm{k}_{0}^{-\delta-|\gamma| \delta_{1}}
$$

From this, using Lemma 6.3, one finally gets

$$
\left|\partial_{x}^{\alpha} d_{k}\right| \lesssim \mathrm{k}_{0}^{-|\alpha| \delta_{1}-\delta} .
$$


Performing the analogous estimates for the $\xi$ derivatives one gets the result.

We are now ready to prove the following:

Lemma 6.7. Let $f \in \mathcal{S F}_{N}^{m}$; then $\langle f\rangle, f^{(r e s)}$ and $f^{(n r)}$ are in $\mathcal{S F}_{N}^{m}$.

Proof. First we remark that, since the cutoffs are functions of $a$ only, Remark 5.11 allows to compute the Fourier coefficients of the different parts of $f$.

Consider $f^{(r e s)}$. Since the seminorms of the cutoffs are bounded uniformly with respect to $k$, one has

$$
\left\|f^{(r e s)}\right\|_{\alpha, \beta, N}^{(m)}=\sum_{k}\left\|\chi_{k} \tilde{\chi}_{k}(1-\chi(a)) \hat{f}_{k}\right\|_{\alpha, \beta}^{(m)}\langle k\rangle^{N} \lesssim \sum_{k}\left\|\hat{f}_{k}\right\|_{\alpha, \beta}^{(m)}\langle k\rangle^{N} \lesssim\|f\|_{\alpha, \beta, N}^{(m)}
$$

so that $f^{(r e s)} \in \mathcal{S} \mathcal{F}_{N}^{m}$.

The other parts of $f$ can be estimated exactly in the same way.

Lemma 6.8. Let $N \epsilon \geq \mathrm{N} \rho$, then one has $f^{(S)} \in \mathcal{S F}_{0}^{m-\mathrm{N} \rho}$.

Proof. First we remark that, since $\chi(\|a\|) f$ has compact support, the thesis trivially holds for this part of $f^{(S)}$. For the other part, it is enough to observe that on $\operatorname{supp}\left(1-\tilde{\chi}_{k}(x, \xi)\right)$ we have

$$
\|k\| \geq(a(x, \xi))^{\epsilon} \gtrsim\left(\mathrm{k}_{0}(x, \xi)\right)^{\epsilon}
$$

by Remark 4.7 , so that one has

$$
\begin{aligned}
\left\|(1-\chi(\|a\|))\left(1-\tilde{\chi}_{k}\right) \hat{f}_{k}\right\|_{\alpha, \beta}^{(m-\epsilon N)} & \lesssim \sup _{\left|\alpha^{\prime}\right| \leq|\alpha|,\left|\beta^{\prime}\right| \leq|\beta|} \sup _{x, \xi} \frac{\left|\partial_{x}^{\alpha^{\prime}} \partial_{\xi}^{\beta^{\prime}}(1-\chi(\|a\|))\left(1-\tilde{\chi}_{k}\right) \hat{f}_{k}\right|}{\mathrm{k}_{0}^{m-\epsilon N}} \\
& =\sup _{\left|\alpha^{\prime}\right| \leq|\alpha|,\left|\beta^{\prime}\right| \leq|\beta|} \sup _{x, \xi} \frac{\left|\partial_{x}^{\alpha^{\prime}} \partial_{\xi}^{\beta^{\prime}}(1-\chi(\|a\|))\left(1-\tilde{\chi}_{k}\right) \hat{f}_{k}\right|}{\mathrm{k}_{0}^{m}} \frac{\mathrm{k}_{0}^{\epsilon N}}{\langle k\rangle^{N}}\langle k\rangle^{N} \\
& \lesssim \sup _{\left|\alpha^{\prime}\right| \leq|\alpha|,\left|\beta^{\prime}\right| \leq|\beta|} \sup _{x, \xi} \frac{\left|\partial_{x}^{\alpha^{\prime}} \partial_{\xi}^{\beta^{\prime}}(1-\chi(\|a\|))\left(1-\tilde{\chi}_{k}\right) \hat{f}_{k}\right|}{\mathrm{k}_{0}^{m}}\langle k\rangle^{N} \\
& \lesssim\left\|\hat{f}_{k}\right\|_{\alpha, \beta}^{(m)}\langle k\rangle^{N}
\end{aligned}
$$

from which, summing over $k$ one gets the thesis. 
Lemma 6.9. Let $f \in \mathcal{S F}_{N}^{m}$ a real valued symbol. Then the equation

$$
\left\{h_{0} ; g\right\}+f^{(n r)}=0
$$

has a real valued solution $g \in \mathcal{S F}_{N}^{m-\delta}$, defined by

$$
g(x, \xi):=\sum_{k \in \mathbb{Z}^{2} \backslash\{0\}} d_{k}(x, \xi) \tilde{\chi}_{k}(x, \xi)(1-\chi(\|a\|)) \hat{f}_{k}(x, \xi) .
$$

Proof. First we verify that $g$ solves 6.13. One has

$$
\left\{h_{0} ; g\right\}=\left.\frac{d}{d t}\right|_{t=0} g \circ \phi_{a}^{\omega t}=\left.\frac{d}{d t}\right|_{t=0} \sum_{k \in \mathbb{Z}^{2}} \hat{g}_{k} e^{\mathrm{i} k \cdot \omega t}=\sum_{k \in \mathbb{Z}^{2}} \mathrm{i}(\omega \cdot k) \hat{g}_{k},
$$

thus, recalling the definition of $f^{(n r)}$, Equation 6.13 reads

$$
\mathrm{i}(\omega \cdot k) \hat{g}_{k}=\tilde{\chi}_{k}\left(1-\chi_{k}\right)(1-\chi(\|a\|)) \hat{f}_{k},
$$

which immediately implies that $g$ defined as in (6.14) solves (6.13). In order to prove that $g \in \mathcal{S F}_{N}^{m-\delta}$, one argues as in the proof of Lemma 6.7, namely since the seminorms of the cutoffs and of $d_{k}$ are bounded uniformly with respect to $k$, one has

$$
\begin{array}{r}
\|g\|_{\alpha, \beta, N}^{(m-\delta)}=\sum_{k}\left\|d_{k} \tilde{\chi}_{k}(1-\chi(\|a\|)) \hat{f}_{k}\right\|_{\alpha, \beta}^{(m-\delta)}\langle k\rangle^{N} \\
\lesssim \sum_{k}\left\|d_{k}\right\|_{\alpha, \beta}^{(-\delta)}\left\|\hat{f}_{k}\right\|_{\alpha, \beta}^{(m)}\langle k\rangle^{N} \lesssim\|f\|_{\alpha, \beta, N}^{(m)}
\end{array}
$$

We are finally able to prove Lemma 6.2.

Proof of the normal form Lemma 6.2. The proof is obtained working inductively. For $n=0$, the thesis holds true with $z_{n}=0$ and $v_{n}=v$; recall indeed that, by Lemma 5.14, $v \in S_{H R}^{m} \subset \mathcal{S F}_{N}^{m}$. We now construct a pseudodifferential operator $G_{n+1}$ with symbol $g_{n+1} \in \mathcal{S} \mathcal{F}_{N}^{m-n \rho-\delta}$ such that $H_{n+1}=$ $e^{\mathrm{i} G_{n+1}} H_{n} e^{-\mathrm{i} G_{n+1}}$. Using the notation 5.21, we have $H_{n+1}=O p^{w}\left(h_{n}^{\prime}+w_{n}^{\prime}\right)$, 
where $w_{n}^{\prime} \in \mathcal{S} \mathcal{F}_{0}^{m-N \rho}$ by Lemma 5.20, and $h_{n}^{\prime}$ is given by

$$
\begin{aligned}
h_{n}^{\prime} & =h_{0}+z^{(n)}+v_{n}+\left\{h_{0} ; g_{n+1}\right\}_{M} \\
& +h_{0}^{\prime}-h_{0}-\left\{h_{0} ; g_{n+1}\right\}_{M}+z^{(n) \prime}-z^{(n)}+v_{n}^{\prime}-v_{n} \\
& =h_{0}+z^{(n)}+v_{n}^{(r e s)}+\left\langle v_{n}\right\rangle+v_{n}^{(S)} \\
& +v_{n}^{(n r)}+\left\{h_{0} ; g_{n+1}\right\} \\
& +\left\{h_{0} ; g_{n+1}\right\}_{M}-\left\{h_{0} ; g_{n+1}\right\} \\
& +h_{0}^{\prime}-h_{0}-\left\{h_{0} ; g_{n+1}\right\}_{M} \\
& +z^{(n) \prime}-z^{(n)} \\
& +v_{n}^{\prime}-v_{n} .
\end{aligned}
$$

We use Lemma 6.9 to construct $g_{n+1}$ in such a way that 6.20 vanishes. Then we define

$$
\begin{aligned}
z_{n+1} & :=\left\langle v_{n}\right\rangle, \quad z^{(n+1)}:=z^{(n)}+v_{n}^{(r e s)}+z_{n+1} \\
& v_{n+1}:=6.21+6.22+6.23+6.24
\end{aligned}
$$

and

$$
w_{n+1}:=w_{n}^{\prime}+v_{n}^{(S)} .
$$

We now study the classes of the different lines. We just compute the order of each term as a symbol in $\mathcal{S F}_{N}$. The order of 6.21) is

$$
\frac{2 \ell}{\ell+1}+m-n \rho-\delta-3\left(\delta_{1}+\delta_{2}\right)=m-n \rho-\rho_{1},
$$

with

$$
\rho_{1}=3\left(\delta_{1}+\delta_{2}\right)+\delta-\frac{2 \ell}{\ell+1}=7(\delta-M)+2 \geq \rho .
$$

To estimate the order of 6.22 we remark that, according to 5.24 it is the same as the order of

$$
\begin{array}{r}
\left\{\left\{h_{0} ; g_{n+1}\right\}_{M} ; g_{n+1}\right\}_{M}=\left\{\left\{h_{0} ; g_{n+1}\right\} ; g_{n+1}\right\}_{M} \\
+\left\{\left\{h_{0} ; g_{n+1}\right\}_{M}-\left\{h_{0} ; g_{n+1}\right\} ; g_{n+1}\right\}_{M}
\end{array}
$$

Now, exploiting the definition of $g_{n+1}$, the r.h.s. of 6.28 is equal to $-\left\{v_{n}^{(n r)} ; g_{n+1}\right\}_{M}$ whose order is

$$
m-n \rho+m-n \rho-\delta-\delta_{1}-\delta_{2}=m-n \rho-\rho_{2}
$$

with

$$
\rho_{2}=\delta+\delta_{1}+\delta_{2}-m \geq \rho
$$


Concerning (6.29), its order is

$$
m-n \rho-\rho_{1}+m-n \rho-\delta-\delta_{1}-\delta_{2}<m-n \rho-\rho_{1} .
$$

Finally the order of $(6.23)$ is $m-n \rho-\rho_{2}$ and the order of $(6.24)$ is the same as the order of $(6.28)$. This concludes the proof.

\section{Spectral result}

In this section we prove the spectral asymptotic (2.20) claimed in Theorem 2.8 .

Definition 7.1. Let $\varsigma, \delta, \epsilon$ fulfilling (2.17) and (2.18), $\mathrm{C}$ as in (2.12), define

$$
\tilde{\Omega}:=\left\{\mathrm{a} \in \mathrm{C}|| \omega(\mathrm{a}) \cdot k \mid \geq 2\|k\|\|\mathrm{a}\|^{\delta} \quad \forall k \in \mathbb{Z}^{2} \text { s.t. } 0<\|k\|<2\|\mathrm{a}\|^{\epsilon}\right\} .
$$

The main result of this section is the following

Theorem 7.2. Given $\mathrm{R}>0$, let

$$
\Omega:=\tilde{\Omega} \cap B_{\mathrm{R}}^{c},
$$

with $\tilde{\Omega}$ as in $(7.1)$. There exist a sequence of symbols $\left\{\tilde{z}_{j}\right\}_{j \geq 1}, \tilde{z}_{j} \in S_{C}^{m-\rho j, \varsigma}$, such that if $\mathrm{R}>0$ is big enough, for any $\mathrm{a} \in \Omega$ there exists an eigenvalue $\lambda_{\mathrm{a}}$ of (2.14) which admits the asymptotic expansion

$$
\lambda_{\mathrm{a}} \sim \tilde{h}_{0}(\mathrm{a})+\sum_{j \geq 0} \tilde{z}_{j}(\mathrm{a})
$$

where $\tilde{h}_{0}(\mathrm{a}) \sim \sum_{j \geq 0} \tilde{h}_{0, j}(\mathrm{a})$, is the function 2.13).

The proof is based on a quasi-mode argument; in particular, our aim is to prove that the joint eigenfunctions of $A_{1}$ and $A_{2}$ defined as in (2.8) are quasi-modes for the normal form operator $H_{\mathrm{N}}$. Since $\mathrm{N}$ is arbitrary, the result follows. The first property we exploit is the following:

Remark 7.3. By the ellipticity of $I d+A_{1}^{2}+A_{2}^{2}$, the joint eigenfunctions $\psi_{\mathrm{a}}$ of $A_{1}$ and $A_{2}$ (defined by (2.8)) satisfy

$$
\left\|\psi_{\mathrm{a}}\right\|_{s} \lesssim s\langle a\rangle^{s}, \quad \forall s \in \mathbb{R}
$$

and therefore, if $R$ is a smoothing operator,

$$
\left\|R \psi_{\mathrm{a}}\right\|_{L^{2}} \lesssim n \frac{1}{\langle a\rangle^{n}}, \quad \forall n \in \mathbb{N} .
$$


The second key property that we exploit for the proof concerns symbols which are functions of the actions only.

First of all, we give the following Lemma which is a variant of Theorem 1 of [Cha83a]:

Lemma 7.4 (Theorem 1 of Cha83a]). Given $m \in \mathbb{R}$ and $0<\varsigma \leq 1$, let $f \in S_{C}^{m, \varsigma}$, there exists a sequence of symbols $\left\{\check{f}_{j}\right\}_{j \in \mathbb{N}}$ with $\check{f}_{j} \in S_{C}^{m-\varsigma j, \varsigma}$ and $\check{f}_{0}=f$ such that $\forall \mathrm{N} \in \mathbb{N}$

$$
f(A)=\sum_{0 \leq j<\mathrm{N}} O p^{w}\left(\check{f}_{j} \circ a\right)+R_{\mathrm{N}}
$$

where $R_{\mathrm{N}}$ is a smoothing operator of order $\varsigma \mathrm{N}-m$ and $f(A)$ is spectrally defined.

Moreover,

(1) $\operatorname{supp}\left(\check{f}_{j}\right) \subseteq \operatorname{supp}(f)$ for all $j$

(2) $\forall s, \exists \alpha, \beta$ and $C_{\mathrm{N}}$, independent of $f$ s.t.

$$
\left\|R_{\mathrm{N}}\right\|_{s, s-(m-\varsigma \mathrm{N})} \leq C_{\mathrm{N}}\|f\|_{\alpha, \beta}^{(m)} .
$$

The proof is easily obtained remarking that in our context formula (3.10) of [Cha83a] holds with $a_{f j} \in S_{C}^{m-j \varsigma, \varsigma}$, which also allows to adapt the estimate of the remainder done in Sect. 4 of that paper.

As a consequence, one has the following:

Lemma 7.5. Given $0<\varsigma \leq 1$ and $f \in S_{C}^{m, \varsigma}$, consider $f \circ a$, then there exists a sequence of symbols $\left\{\tilde{f}_{j}\right\}_{j \in \mathbb{N}}$ with $\tilde{f}_{j} \in S_{C}^{m-\varsigma j, \varsigma} \forall j$ and $\tilde{f}_{0}=f$ such that for any $n \in \mathbb{N}$

$$
O p^{w}(f \circ a)=\sum_{0 \leq j<\mathrm{N}} \tilde{f}_{j}(A)+R_{\mathrm{N}}^{\prime}
$$

where $R_{\mathrm{N}}^{\prime}$ is a smoothing operator of order $\varsigma \mathrm{N}-m$. Moreover,

(1) $\operatorname{supp}\left(\tilde{f}_{j}\right) \subseteq \operatorname{supp}(f) \forall j$

(2) $\exists \alpha, \beta$ and $C_{\mathrm{N}}$, independent of $f$ s.t.

$$
\left\|R_{\mathrm{N}}^{\prime}\right\|_{s, s-(m-\varsigma \mathrm{N})} \leq C_{\mathrm{N}}\|f\|_{\alpha, \beta}^{(m)} .
$$

In what follows we will denote $\tilde{f} \sim \sum_{j \geq 0} \tilde{f}_{j}$. 
Proof. The proof is obtained arguing inductively. In particular, fix $\mathbb{N} \in \mathbb{N}$ : we prove that for any $\bar{n} \leq \mathrm{N}$ there exist $\tilde{f}_{0}, \ldots, \tilde{f}_{\bar{n}}$, with $\tilde{f}_{j} \in S_{C}^{m-\varsigma j, \varsigma} \forall j$, such that

$$
O p^{w}(f \circ a)-\sum_{0 \leq j<\bar{n}} \tilde{f}_{j}(A)=O p^{w}\left(\tau^{(\bar{n})} \circ a\right)+R_{\bar{n}}^{\prime}
$$

where $\tau^{(\bar{n})} \in S_{C}^{m-\varsigma(\bar{n}+1), \varsigma}$ and $R_{\bar{n}}^{\prime}$ is a smoothing operator of order $\varsigma \mathrm{N}-m$. If $\bar{n}=0$, Lemma 7.4 implies that $(7.6)$ is satisfied with $\tilde{f}_{0}=f$. Indeed, one has

$$
O p^{w}(f \circ a)-\tilde{f}_{0}(A)=O p^{w}(f \circ a)-f(A)=-\sum_{1 \leq j<\mathrm{N}} O p^{w}\left(f_{j} \circ a\right)-R_{\mathrm{N}},
$$

which implies (7.6) with $\tau^{(0)}:=-\sum_{1 \leq j<\mathrm{N}} f_{j} \in S_{C}^{m-\varsigma, \varsigma}$ and $R_{0}^{\prime}:=R_{\mathrm{N}}$.

Suppose now that $(7.6)$ is satisfied for some $\bar{n} \geq 0$; then one chooses $\tilde{f}^{(\bar{n}+1)}=-\tau^{(\bar{n})}$ and, again by Lemma 7.4 , obtains

$$
\begin{aligned}
O p^{w}(f \circ a)-\sum_{0 \leq j<\bar{n}+1} \tilde{f}_{j}(A) & =O p^{w}(f \circ a)-\sum_{0 \leq j<\bar{n}} \tilde{f}_{j}(A)+\tau^{(j)}(A) \\
& =O p^{w}\left(\tau^{(\bar{n})} \circ a\right)+R_{\bar{n}}^{\prime}-\sum_{0 \leq j \leq \mathrm{N}} O p^{w}\left(\tau_{j}^{(\bar{n})} \circ a\right)+R_{\bar{n}},
\end{aligned}
$$

with $R_{\bar{n}}$ a smoothing operator of order $\varsigma \mathrm{N}-m$. Thus $(7.6)$ is satisfied at the step $\bar{n}+1$, with $R_{\bar{n}+1}^{\prime}=R_{\bar{n}}^{\prime}-R_{\bar{n}}$ and $\tau^{(\bar{n}+1)}=-\sum_{1 \leq j \leq \mathrm{N}} \tau_{j}^{(\bar{n})}$.

By the above Lemma $\psi_{\mathrm{a}}$ is a quasimode for $O p^{w}(f \circ a)$, so one immediately gets the following Lemma

Lemma 7.6. Fix $m \in \mathbb{R}$ and $0<\varsigma \leq 1$. Suppose $F=O p^{w}(f)$ is a selfadjoint operator whose symbol $f \in S_{A N, \delta}^{m}$ is a function of the actions only. Then there exists a sequence $\tilde{f}_{j} \in S_{C}^{m-\varsigma j, \varsigma}$ of functions with $\tilde{f}_{0}=f_{c}$ such that, for any $\mathrm{a} \in\left(\mathbb{Z}^{2}+\kappa\right) \cap \mathcal{C}$ sufficiently large, there exists an eigenvalue $\lambda_{\mathrm{a}}$ of $F$ fulfilling

$$
\lambda_{\mathrm{a}} \sim \tilde{f}(\mathrm{a})=\sum_{j \geq 0} \tilde{f}_{j}(\mathrm{a}) .
$$

Proof. Let $\Psi \in S_{C}^{0, \varsigma}$ be a cutoff function equal to 1 on $\mathcal{C}$, with support contained in $\mathcal{V}$ which is homogeneous of degree zero. By Lemmas 7.45 .12 , and 7.5, for $a \in C$ sufficiently large one has

$$
\begin{aligned}
O p^{w}(f) \psi_{\mathrm{a}} & =O p^{w}(f)(1-\chi(\|A\|)) \Psi(A) \psi_{\mathrm{a}} \\
& =O p^{w}(f \sharp(1-\check{\chi}(\|a\|)) \sharp \check{\Psi}(a)) \psi_{\mathbf{a}}+R_{N} \psi_{\mathrm{a}} \\
& =O p^{w}\left(f_{c} \sharp(1-\check{\chi}(\|a\|)) \sharp \check{\Psi} \circ a\right) \psi_{\mathbf{a}}+R_{N} \psi_{\mathrm{a}} \\
& =O p^{w}\left(f_{c}(a)\right)((1-\chi(\|A\|))) \Psi(A) \psi_{\mathbf{a}}+R_{N} \psi_{\mathrm{a}} \\
& =O p^{w}\left(f_{c}(a)\right) \psi_{\mathbf{a}}+R_{N} \psi_{\mathbf{a}}=\tilde{f}_{c}(\mathrm{a}) \psi_{\mathbf{a}}+R_{N} \psi_{\mathbf{a}},
\end{aligned}
$$


with $R_{N}$ a regularizing operator which changes from line to line. From this equation, by a quasimode argument the thesis immediately follows (in the statement, we just omitted the index $c$ from $\tilde{f}$ ).

We come to the proof of Theorem 7.2 .

Proof of Theorem 7.2. We apply again a quasimode argument to $H_{\mathrm{N}}$. We start with observing that, up to a pseudo-differential operator of order $m-\mathrm{N} \rho$, $H_{\mathrm{N}}$ has symbol given by

$$
f+z_{\mathrm{N}}^{(r e s)}, \quad f:=h_{0}(a)+\sum_{j=1}^{\mathrm{N}} z_{j}(a) .
$$

With the notations of Lemma 7.6, we aim at proving that, for a $\in \Omega$ sufficiently large,

$$
\left\|H_{\mathrm{N}} \psi_{\mathrm{a}}-\left(\tilde{h}_{0}(\mathrm{a})+\sum_{j=1}^{\mathrm{N}} \tilde{z}_{j}(\mathrm{a})\right) \psi_{\mathrm{a}}\right\| \lesssim_{\mathrm{N}}\|\mathrm{a}\|^{m-\mathrm{N} \rho},
$$

so that there exists an eigenvalue $\lambda_{\mathrm{a}}$ of $H_{\mathrm{N}}$ fulfilling

$$
\left|\lambda_{\mathrm{a}}-\tilde{h}_{0}\left(\lambda_{\mathrm{a}}\right)-\sum_{j=1}^{\mathrm{N}} \tilde{z}_{j}\left(\lambda_{\mathrm{a}}\right)\right| \lesssim_{\mathrm{N}}\|\mathrm{a}\|^{m-\mathrm{N} \rho} .
$$

First we focus on the normal form term $z_{\mathrm{N}}^{(r e s)}$. Define

$$
\eta_{k}(a):=\chi\left(\frac{\omega(a) \cdot k}{2\|k\|\|a\|^{\delta}}\right)\left(1-\chi\left(\frac{\|k\|}{2\|a\|^{\epsilon}}\right)\right) ;
$$

then, arguing as in Lemmas 6.3 and 6.4 we have that $\eta_{k}(a) \in S_{A N, \delta}^{0}$, with seminorms that are uniformly bounded in $k$, and

$$
\begin{gathered}
z_{\mathrm{N}}^{(\text {res })}=\sum_{k \neq 0} \hat{z}_{\mathrm{N}, k}^{(\text {res })}=\sum_{k \neq 0} \hat{z}_{\mathrm{N}, k}^{(\text {res })} \eta_{k}=\sum_{k \neq 0}\left(\hat{z}_{\mathrm{N}, k}^{(\text {res })} \sharp \eta_{k}+r_{\mathrm{N}, k}^{(2)}\right), \\
r_{\mathrm{N}, k}^{(2)} \in S_{A N, \delta}^{-\bar{n}} \quad \forall \bar{n} \in \mathbb{N},
\end{gathered}
$$

where the second equality in 7.11 is due to the fact that $\eta=1$ on the support of $\hat{z}_{\mathrm{N}, k}^{(r e s)}$, and the third equality is due to Lemma 5.4 and to the fact that also the derivatives of $\eta$ vanish on the support of $\hat{z}_{\mathrm{N}, k}^{(\text {res })}$.

It follows that

$$
\begin{gathered}
O p^{w}\left(z_{\mathrm{N}}^{(r e s)}\right)=\sum_{k}\left(O p^{w}\left(\hat{z}_{k}\right) O p^{w}\left(\eta_{k}\right)+R_{\mathrm{N}, k}^{(2)}\right) \\
=\sum_{k}\left(O p^{w}\left(\hat{z}_{k}\right)\left(\tilde{\eta}_{k}(A)+R_{\mathrm{N}, k}^{(3)}\right)+R_{\mathrm{N}, k}^{(2)}\right)
\end{gathered}
$$


with $\tilde{\eta}_{k}$ a function with the same support of $\eta_{k}$ and with uniformly bounded norms and $R_{\mathrm{N}, k}^{(2)}, R_{\mathrm{N}, k}^{(3)}$ smoothing operator with norms which are respectively summable and uniformly bounded in $k$, due to estimates (5.8) and (7.5) respectively. By the very definition of the set $\Omega$, one has $\eta(A) \psi_{\mathrm{a}}=0$ for any $\mathrm{a} \in \Omega$, thus

$$
z_{\mathrm{N}}^{(r e s)} \psi_{\mathrm{a}}=R_{\mathrm{N}}^{\prime} \psi_{\mathrm{a}}
$$

for some smoothing operator $R_{\mathrm{N}}^{\prime}$. Then combining (7.12) and equation (7.8), with $f=h_{0}(a)+\sum_{j=1}^{\mathbb{N}} z_{j}(a)$, we obtain that there exists $R_{\mathbb{N}} \in S_{A N, \delta}^{m-\mathbb{N} \rho}$ such that

$$
H_{\mathrm{N}} \psi_{\mathrm{a}}=\left(\tilde{h}_{0}(\mathrm{a})+\sum_{j=1}^{\mathrm{N}} \tilde{z}_{j}(\mathrm{a})\right) \psi_{\mathrm{a}}+R_{\mathrm{N}} \psi_{\mathrm{a}},
$$

which implies (7.9).

\section{Cardinality estimates}

\subsection{Nondegenerate homogeneous frequency maps}

In this section we prove that the non resonant set $\Omega$ defined in $(7.2)$ is of density one in $\mathrm{C}$, thus concluding the proof of Theorem 2.8. More precisely, we prove that the complementary of $\Omega$ has density zero.

The strategy consists in reducing the estimate of the cardinality of sets to measure estimates. Then we have to estimate the measure of resonant sets. To this end we exploit the homogeneity of the nonresonance condition with respect to $a$ in order to reduce the estimate of their measure to a measure estimate on the intersection of $\mathcal{C}$ with the unit sphere. Finally, the estimate on the unit sphere is done exploiting the tools developed in the context of degenerate KAM theory, in particular by Rüssmann.

First of all, define

$$
\Gamma:=\mathbb{Z}^{2}+\kappa
$$

We start by defining the resonant sets and the "cutoff sets".

$$
\begin{array}{r}
\Sigma_{k}(\gamma):=\left\{a \in \mathcal{C}:|\omega(a) \cdot k| \leq \gamma\|k\|\|a\|^{\delta}\right\} \\
\mathcal{T}_{k}(\gamma):=\left\{a \in \mathcal{C}:\|k\|<\gamma\|a\|^{\epsilon}\right\} \\
\Sigma(\gamma):=\bigcup_{k \in \mathbb{Z}^{2} \backslash\{0\}}\left(\Sigma_{k}(2) \cap \mathcal{T}_{k}(2)\right) .
\end{array}
$$

The main result of this section is the following Theorem. 
Theorem 8.1. Assume $\delta>M-1$, then $\exists C>0, \mu_{0} \in \mathbb{N}$ s.t. for $R$ large enough, one has

$$
\#\left(\Gamma \cap \Sigma(\gamma) \cap B_{R}\right) \leq C \gamma^{1 / \mu_{0}} \frac{R^{2}}{R^{\frac{M-\delta}{\mu_{0}}-2 \epsilon}} .
$$

The rest of the section is devoted to the proof of this theorem.

First, we fix a large $R_{0}$ and we will work in the ball in the action space centered at the origin and with radius $R$ larger than $R_{0}: R \geq R_{0}$. In the following we will assume that $R_{0}$ is as large as needed. Following [BLM20b], given $r \in \mathbb{R}^{+}$and a set $\mathcal{A}$, we define

$$
\mathcal{A}^{(r)}:=\bigcup_{\mathrm{a} \in \mathcal{A}} B_{r}(\mathrm{a})
$$

so that we have the following remark

Remark 8.2. [Remark 5.12 of [BLM20b]] Let $\mathcal{A}$ be a set and let $r<1 / 2$, then

$$
\#(\mathcal{A} \cap \Gamma) \leq \frac{\left|\mathcal{A}^{(r)}\right|}{\left|B_{r}\right|} .
$$

\section{Lemma 8.3.}

$$
\mathcal{T}_{k}^{(r)}(2) \subset \mathcal{T}_{k}(1)
$$

Proof. By definition

$$
\mathcal{T}_{k}^{(r)}(2)=\left\{a: \exists \tilde{a}:\|a-\tilde{a}\| \leq r ;\|\tilde{a}\|^{\epsilon} \geq 2\|k\|\right\} .
$$

We study $\|a\|$. One has

$$
\|a\| \geq\|\tilde{a}\|-\|a-\tilde{a}\| \geq 2\|k\|^{1 / \epsilon}-r=\|k\|^{1 / \epsilon}\left(2-\frac{r}{\|k\|^{1 / \epsilon}}\right),
$$

but the parenthesis is larger than 1 , as it is easy to verify using $r<1 / 2$.

Lemma 8.4. Define

$$
C:=\sup _{\|a\|=1, a \in \mathcal{C}}\|d \omega(a)\|
$$

assume

$$
\|a\| \geq \frac{1}{2}\left(\frac{2 r C}{\gamma}\right)^{\frac{1}{\delta+1-M}}
$$

then

$$
\Sigma_{k}^{(r)}(\gamma) \subset \Sigma_{k}(\tilde{\gamma}), \quad \tilde{\gamma}:=\frac{\gamma}{2^{\delta+1}}
$$


Proof. We denote $a=\lambda u$, with $\lambda=\|a\|$ and $u \in \mathcal{C}$, and similarly $\tilde{a}=\tilde{\lambda} \tilde{u}$ and so on. Let $a \in \Sigma_{k}^{(r)}(\gamma)$, then there exists $\bar{a} \in \Sigma_{k}(\gamma)$ s.t. $\|a-\bar{a}\| \leq r$, thus one has

$$
\left|\frac{\omega(a) \cdot k}{\|k\|}\right| \geq\left|\frac{\omega(\bar{a}) \cdot k}{\|k\|}\right|-\left|\frac{d(\omega(\tilde{a}) \cdot k)(a-\bar{a})}{\|k\|}\right|,
$$

with some $\tilde{a}$, fulfilling $\frac{\|a\|}{2} \leq\|\tilde{a}\| \leq 2\|a\|$. Of course the same inequality is true if one replaces $\bar{a}$ to $\tilde{a}$. So we have

$$
\left|\frac{d(\omega(\tilde{a}) \cdot k)(a-\bar{a})}{\|k\|}\right| \leq C \tilde{\lambda}^{M-1} r \leq C 2^{M-1} \lambda^{M-1} r,
$$

and also

$$
\left|\frac{\omega(\tilde{a}) \cdot k}{\|k\|}\right| \geq \tilde{\lambda}^{\delta} \gamma \geq \frac{\lambda^{\delta} \gamma}{2^{\delta}}
$$

If 8.4 is satisfied then 8.5 is smaller than a half of 8.6 and implies

$$
\left|\frac{\omega(a) \cdot k}{\|k\|}\right| \geq \frac{\lambda^{\delta} \gamma}{2^{\delta+1}}
$$

which is the thesis.

We are now going to estimate the measure of $\Sigma_{k}\left(\gamma / 2^{\delta+1}\right) \cap \mathcal{T}_{k}(1) \cap B_{R}$. To this end we exploit the homogeneity of the frequencies. We denote

$$
S_{\lambda}:=\left\{a \in \mathbb{R}^{2}:\|a\|=\lambda\right\},
$$

and we will exploit the following

\section{Remark 8.5.}

$$
\left|\Sigma_{k}(\tilde{\gamma}) \cap \mathcal{T}_{k}(1) \cap B_{R} \cap B_{R_{0}}^{c}\right|=\int_{R_{0}}^{R}\left|\Sigma_{k}(\tilde{\gamma}) \cap \mathcal{T}_{k}(1) \cap S_{\lambda}\right| d \lambda .
$$

In order to estimate the above quantity we establish the following Lemma

\section{Lemma 8.6.}

$$
\Sigma_{k}(\tilde{\gamma}) \cap \mathcal{T}_{k}(1) \cap S_{\lambda}=\left\{\begin{array}{cl}
\emptyset & \text { if } \lambda^{\epsilon} \leq\|k\| \\
\lambda\left(\Sigma_{k}\left(\frac{\tilde{\gamma}}{\lambda^{M}-\delta}\right) \cap S_{1}\right) & \text { if } \lambda^{\epsilon}>\|k\|
\end{array},\right.
$$

where the multiplication of a set by $\lambda$ means multiplication of each one of its elements. 
Proof. Just remark that (with $u=a / \lambda$ ), we have

$$
\Sigma_{k}(\tilde{\gamma}) \cap S_{\lambda}=\left\{a=\lambda u: u \in S_{1} \text { and }\left|\frac{\omega(\lambda u) \cdot k}{\|k\|}\right| \geq \lambda^{\delta} \tilde{\gamma}\right\},
$$

but the nonresonance condition can be rewritten using the homogeneity of $\omega$ as

$$
\left|\frac{\omega(u) \cdot k}{\|k\|}\right| \geq \lambda^{\delta-M} \tilde{\gamma} .
$$

In order to conclude the proof just remark that the intersection with $\mathcal{T}_{k}(1)$ is empty or full according to the conditions in the Lemma.

We are now in the position of using degenerate KAM theory in order to estimate $\Sigma_{k}\left(\frac{\tilde{\gamma}}{\lambda^{M}-\delta}\right) \cap S_{1}$. First we recall a couple of lemmas and definitions from [Rüs01] (see also [BBM11]).

First we adapt the notation. Thus, consider the functions $\left(\omega_{1}(a), \omega_{2}(a)\right)$, and restrict them to the intersection of the set $\Pi$ with the unit sphere. Precisely, consider

$$
\begin{array}{r}
\omega(\phi) \equiv\left(\omega_{1}(\phi), \omega_{2}(\phi)\right) \\
\omega_{j}(\phi):=\omega_{j}(\cos \phi, \sin \phi), \quad \phi \in\left[0, \frac{3}{4} \pi\right] .
\end{array}
$$

Definition 8.7. The function $\left(\omega_{1}(\phi), \omega_{2}(\phi)\right)$ is said to be weakly nondegenerate if $\forall\left(c_{1}, c_{2}\right) \neq(0,0)$ the function

$$
c_{1} \omega_{1}(\phi)+c_{2} \omega_{2}(\phi)
$$

is not identically zero.

We will prove below (see Lemma 8.13 ) that $\omega(\phi)$ is analytic on a complex neighborhood of the interval $[0,3 / 4 \pi]$ and that it is weakly nondegenerate.

Lemma 8.8. Assume that $\omega$ is weakly nondegenerate, then there exist $\beta>0$ and $1 \leq \mu_{0} \in \mathbb{N}$ s.t.

$$
\max _{0 \leq \mu \leq \mu_{0}}\left|\frac{d^{\mu}}{d \phi^{\mu}} \frac{k \cdot \omega(\phi)}{\|k\|}\right| \geq \beta, \quad \forall \phi \in[0,3 / 4 \pi], \quad \forall k \in \mathbb{Z}^{2} \backslash\{0\} .
$$

Proof. By contradiction: assume that $\forall \mu_{0}$ and $\forall \beta>0 \exists \phi_{\mu_{0}, \beta}, k_{\mu_{0}, \beta}$ s.t.

$$
\max _{0 \leq \mu \leq \mu_{0}}\left|\frac{d^{\mu}}{d \phi^{\mu}} \frac{k_{\mu_{0}, \beta} \cdot \omega\left(\phi_{\mu_{0}, \beta}\right)}{\left\|k_{\mu_{0}, \beta}\right\|}\right|<\beta .
$$


Take $\sigma:=\mu_{0}, \beta:=(\sigma+1)^{-1}$, then $\exists \phi_{\sigma}, k_{\sigma}$ s.t.

$$
\max _{0 \leq \mu \leq \mu_{0}}\left|\frac{d^{\mu}}{d \phi^{\mu}} \frac{k_{\sigma} \cdot \omega\left(\phi_{\sigma}\right)}{\left\|k_{\sigma}\right\|}\right|<\frac{1}{\sigma+1} .
$$

But this means that $\forall \mu, \exists \sigma \geq \mu$ s.t.

$$
\left|\frac{d^{\mu}}{d \phi^{\mu}} \frac{k_{\sigma} \cdot \omega\left(\phi_{\sigma}\right)}{\left\|k_{\sigma}\right\|}\right|<\frac{1}{\sigma+1} .
$$

Take the limit $\sigma \rightarrow \infty$. By compactness $\phi_{\sigma} \rightarrow \bar{\phi}$ and $\frac{k_{\sigma}}{\left\|k_{\sigma}\right\|} \rightarrow \bar{c}=\left(\bar{c}_{1}, \bar{c}_{2}\right)$. Thus taking the limit of (8.11), one gets

$$
\left|\frac{d^{\mu}}{d \phi^{\mu}} \bar{c} \cdot \omega(\bar{\phi})\right|=0 \text {. }
$$

But, by analyticity, this means $\bar{c} \cdot \omega(\bar{\phi}) \equiv 0$, against the assumption of weakly nondegeneracy.

We now recall the following theorem which is a simplification of a theorem by Rüssmann. For the very technical proof we make reference to the original paper

Theorem 8.9. [Theorem 17.1 of [Rüs01]] Let $\mathcal{I} \subset \mathbb{R}$ be compact and denote by $|\mathcal{I}|$ its length. Denote (as above)

$$
\mathcal{I}^{(r)}:=\bigcup_{\phi \in \mathcal{I}} B_{r}(\phi)
$$

Let $g \in C^{\mu_{0}+1}\left(\mathcal{I}^{(r)}\right)$ be s.t.

$$
\min _{\phi \in \mathcal{I}} \max _{0 \leq \mu \leq \mu_{0}}\left|\frac{d^{\mu} g}{d \phi^{\mu}}(\phi)\right| \geq \beta .
$$

Then $\forall \epsilon>0$

$$
|\{\phi \in \mathcal{I}:|g(\phi)| \leq \epsilon\}| \leq C|\mathcal{I}|\left(\frac{\epsilon}{\beta}\right)^{\frac{1}{\mu_{0}}} \frac{1}{\beta}|g|_{C^{\mu_{0}+1}\left(\mathcal{I}^{(r)}\right)} .
$$

In the original version the constant $C$ is explicitly computed, but here we do not need its value.

Corollary 8.10. $\exists \mu_{0}, C>0$ s.t.

$$
\left|\Sigma_{k}(\gamma) \cap S_{1}\right| \leq C \gamma^{1 / \mu_{0}}, \forall k \in \mathbb{Z}^{2} \backslash\{0\} .
$$


Using this corollary we prove now the following Lemma.

Lemma 8.11. There exist $C>0$ such that, if $R>R_{0}$,

$$
\left|\bigcup_{k \neq 0}\left(\Sigma_{k}(\tilde{\gamma}) \cap \mathcal{T}_{k}(1)\right) \cap B_{R}\right| \leq C \frac{R^{2}}{R^{\frac{M-\delta}{\mu_{0}}-2 \epsilon}}
$$

Proof. Define $B_{R, R_{0}}:=B_{R} \cap B_{R_{0}}^{c}$ and just observe that

$$
\begin{aligned}
\left|\bigcup_{k \neq 0}\left(\Sigma_{k}(\tilde{\gamma}) \cap \mathcal{T}_{k}(1)\right) \cap B_{R}\right| & =\left|\bigcup_{k \neq 0}\left(\Sigma_{k}(\tilde{\gamma}) \cap \mathcal{T}_{k}(1)\right) \cap B_{R_{0}}\right| \\
& +\left|\bigcup_{k \neq 0}\left(\Sigma_{k}(\tilde{\gamma}) \cap \mathcal{T}_{k}(1)\right) \cap B_{R, R_{0}}\right| \\
& \leq 2\left|\bigcup_{k \neq 0}\left(\Sigma_{k}(\tilde{\gamma}) \cap \mathcal{T}_{k}(1)\right) \cap B_{R, R_{0}}\right|
\end{aligned}
$$

if $R$ is big enough, thus

$$
\begin{aligned}
\bigcup_{k \neq 0}\left(\Sigma_{k}(\tilde{\gamma}) \cap \mathcal{T}_{k}(1)\right) \cap B_{R} \mid & \leq 2 \int_{R_{0}}^{R}\left|\bigcup_{k}\left(\Sigma_{k}(\tilde{\gamma}) \cap \mathcal{T}_{k}(1) \cap S_{\lambda}\right)\right| d \lambda \\
& =2 \int_{R_{0}}^{R}\left|\bigcup_{|k| \leq 2 \lambda^{\epsilon}}\left(\Sigma_{k}(\tilde{\gamma}) \cap S_{\lambda}\right)\right| d \lambda \\
& =2 \int_{R_{0}}^{R} \lambda\left|\bigcup_{|k| \leq \lambda^{\epsilon}}\left(\Sigma_{k}\left(\frac{\tilde{\gamma}}{\lambda^{M-\delta}}\right) \cap S_{1}\right)\right| d \lambda \\
& \leq 8 \int_{R_{0}}^{R} \lambda^{1+2 \epsilon} \sup _{k}\left|\left(\Sigma_{k}\left(\frac{\tilde{\gamma}}{\lambda^{M-\delta}}\right) \cap S_{1}\right)\right| d \lambda \\
& \leq C \int_{R_{0}}^{R} \lambda^{1+2 \epsilon}\left(\frac{\tilde{\gamma}}{\lambda^{M-\delta}}\right)^{1 / \mu_{0}} d \lambda \\
& \leq C \frac{R^{2}}{R^{\frac{M-\delta}{\mu_{0}}-2 \epsilon}} .
\end{aligned}
$$

Remark 8.12. By Lemma 8.11 one immediately deduces that the set $\tilde{\Omega}$ defined in (7.1) has density one at infinity, provided $\epsilon$ and $\delta$ are such that $2 \epsilon \mu_{0}<M-\delta$. Thus the same holds true also for the set $\Omega=\tilde{\Omega} \cap B_{\mathrm{R}}^{c}$ defined in 7.2 . 


\subsection{Nondegeneracy of the frequency map of the anhar- monic oscillator}

In this section we prove that the restriction of the frequency map to the unit sphere is weakly nondegenerate.

Actually the proof is a simple consequence of the techniques developed in [FK04, BF17]), here we give it for the sake of completeness.

Lemma 8.13. The function $\left(\omega_{1}, \omega_{2}\right)$ is analytic in a neighborhood of $[0,3 / 4 \pi]$ and is nondegenerate.

Proof. Analyticity in the open set $(0,3 / 4 \pi)$ follows from the fact that the Hamiltonian is an analytic function of the actions on the interior of $\Pi$. Then remark that the points $0,3 / 4 \pi$ correspond to $a_{r}=0$, which is the circular orbit. We consider just the case of $a_{2}>0$, the other one follows easily. $\omega(0)$ is the limit as $\phi \rightarrow 0$ of $\omega(\phi)$. Now, $\omega_{2}$ just converges to the frequency of the circular orbit, while $\omega_{1}$ converges to the frequency of small oscillation of the effective system with Hamiltonian

$$
h_{0}\left(r, p_{r}, a_{2}\right):=\frac{p_{r}^{2}}{2}+\frac{a_{2}^{2}}{2 r^{2}}+\frac{r^{2 \ell}}{2 \ell} .
$$

Now it is well known that the Birkhoff normal form at the minimum of the effective potential (namely the circular orbit) is convergent, and this allows to compute the development of the Hamiltonian, as a function of the actions, at the circular orbit. In particular it turns out that also the frequencies can be extended to complex analytic functions close to the circular orbit.

It was proved in [FK04] that close to the circular orbit the Hamiltonian admits the expansion (for $a_{1} \ll\left|a_{2}\right|$ )

$$
h_{0}\left(a_{1}, a_{2}\right)=V^{*}\left(a_{2}\right)+\sqrt{A\left(a_{2}\right)} a_{1}+\frac{-5 B\left(a_{2}\right)^{2}+3 C\left(a_{2}\right) A\left(a_{2}\right)}{48 A\left(a_{2}\right)^{2}} a_{1}^{2}+o\left(a_{1}^{2}\right),
$$

where

$$
\begin{array}{rlrl}
V^{*}\left(a_{2}\right) & =\frac{a_{2}^{2}}{2 r_{0}^{2}}+V\left(r_{0}\right), & & A\left(a_{2}\right)=\frac{3 a_{2}^{2}}{r_{0}^{4}}+V^{\prime \prime}\left(a_{0}\right), \\
B\left(a_{2}\right) & =-\frac{12 a_{2}^{2}}{r_{0}^{5}}+V^{\prime \prime \prime}\left(r_{0}\right), & C\left(a_{2}\right)=\frac{60 a_{2}^{2}}{r_{0}^{6}}+V^{(4)}\left(r_{0}\right), \\
r_{0}:=a_{2}^{\frac{1}{\ell+1}} . &
\end{array}
$$

We recall that, to obtain this formula, one has to think of $a_{1}$ as $\frac{\tilde{p}_{r}^{2}+\tilde{r}^{2}}{2}$ where $\tilde{p}_{r}$ and $\tilde{r}$ are rescaled, translated variables. An explicit computation gives

$$
h_{0}=\frac{\ell+1}{2 \ell} a_{2}^{\frac{2 \ell}{\ell+1}}+\sqrt{2(\ell+1)} a_{2}^{M} a_{1}+\frac{1}{2} \frac{3 a c-5 b^{2}}{24 a^{2}} a_{2}^{-\frac{2}{\ell+1}} a_{1}^{2}+\text { h.o.t. }
$$


where

$$
a=2(\ell+1), \quad b:=2\left(2 \ell^{2}-2 \ell-5\right), \quad c:=2\left(4 \ell^{3}-12 \ell^{2}+11 \ell+27\right) .
$$

(We emphasize that these letters will be used to denote such quantities only in this proof). From 8.23), one can compute $\omega_{j}=\frac{\partial h}{\partial a_{j}}$ close to any point of the form $\left(a_{1}, a_{2}\right)=\left(0, \bar{a}_{2}\right)$, from which one sees that it extends to a complex analytic function in a neighborhood of such point. One can also compute $\omega_{2} / \omega_{1}$, getting (with some work)

$$
\begin{aligned}
& \omega_{1} \simeq \sqrt{2(\ell+1)} a_{2}^{M}\left(1+\frac{3 a c-5 b^{2}}{24 a^{2} \sqrt{2(\ell+1)}} \frac{a_{1}}{a_{2}}\right), \\
& \omega_{2} \simeq a_{2}^{M}\left(1+M \sqrt{2(\ell+1)} \frac{a_{1}}{a_{2}}\right), \\
& \frac{\omega_{2}}{\omega_{1}} \simeq \frac{1}{\sqrt{2(\ell+1)}}\left(1+\left(M \sqrt{2(\ell+1)}-\frac{3 a c-5 b^{2}}{24 a^{2} \sqrt{2(\ell+1)}}\right) \frac{a_{1}}{a_{2}}\right) .
\end{aligned}
$$

In particular, from (8.24) and 8.25$)$, we notice that both $\omega_{1}$ and $\omega_{2}$ do not vanish for $a_{2} \neq 0$ and $0<a_{1} \ll\left|a_{2}\right|$.

A further computation gives the value of the coefficient of $a_{1} / a_{2}$ in (8.26), which turns our to be

$$
d:=\frac{16(\ell+1)^{2}(\ell-1)(2 \ell+1)}{24 a^{2} \sqrt{2(\ell+1)}} .
$$

So, such a coefficient vanishes only if

$$
\ell=-1 / 2,1,-1
$$

By the way, we remark that they correspond to the Kepler and the Harmonic cases, and also to the degenerate case of potential proportional to $r^{-2}$.

So, if $c_{2} \neq 0, c_{1} \omega_{1}+c_{2} \omega_{2}$ vanishes only if

$$
c_{1}+c_{2}\left(\frac{1}{\sqrt{2(\ell+1)}}\left(1+d \frac{a_{1}}{a_{2}}\right)\right)=0,
$$

but this is a nontrivial analytic function of $a_{1} / a_{2}$, therefore it is also a nontrivial function of $\phi$. If $c_{2}=0$ then $c_{1} \neq 0$, and therefore the same is true, since $\omega_{1}$ is different from zero. 


\section{A Proof of Lemma 4.5}

We start by proving the following easy lemma

Lemma A.1. The function $a_{r}$ defined by 4.4 is analytic on the domain

$$
0<|L|<\left(\frac{2 \ell}{\ell+1} E\right)^{\frac{\ell+1}{2 \ell}}, \quad E>0 ;
$$

Proof. The effective Hamiltonian $h_{0}^{*}\left(r, p_{r}, L\right)$ defined by (4.3) is an analytic function except at $r=0$. Furthermore, for $L \neq 0, h^{*}$, as a function of $r, p_{r}$ is a submersion, except on the level surface of level $E=|L|^{\frac{\ell+1}{2 \ell}}$. So, outside this domain the level surface depend analytically on both $E$ and $L$. Since $a_{r}$ is just the normalized area contained in the level surface, it also depends analytically on $E, L$ in the considered domain.

We now study the behavior of $a_{r}$ as $L \rightarrow 0$. To this end we apply the method of the residues to the integral defining it. Precisely, we prove the following Lemma

Lemma A.2. Define

$$
\rho=: \frac{L^{2}}{E^{\frac{(\ell+1)}{\ell}}},
$$

then there exists $\rho_{*}$ and a function $f(E, L)$, analytic in the domain

$$
E>0, \quad 0 \leq \rho<\rho_{*},
$$

s.t.

$$
a_{r}=-\frac{1}{2}|L|+f(E, L) .
$$

Proof. Performing the change of variables $r^{2}=s$, in the integral defining $a_{r}$ one has

$$
a_{r}=\frac{\sqrt{2}}{2 \pi} \int_{s_{m}}^{s_{M}} \frac{1}{s} \sqrt{-p(s, E, L)} d s,
$$

with

$$
p(s, E, L)=\frac{s^{\ell+1}}{2 \ell}-E s+\frac{L^{2}}{2}
$$

and $s_{m}=s_{m}(E, L), s_{M}=s_{M}(E, L)$ the two positive solutions of the equation $p(s, E, L)=0$.

We now study the zeroes of $p(s, E, L)$. To this aim we observe that, since $p$ is a polynomial in $s$ with coefficients depending on $E$ and $L$, it 
has $\ell+1$ complex roots which of course depend continuously on $E$ and $L$. Actually there is more structure: indeed $s_{*}(E, L)$ is a root of $p$ if and only if $t_{*}=s_{*} E^{-1 / \ell}$ is a root of

$$
\tilde{p}(t)=\frac{t^{\ell+1}}{2 \ell}-t+\frac{1}{2} \rho,
$$

which is a function of $\rho$ only.

Since we are interested in a neighborhood of $\rho=0$, we start by remarking that, for $\rho=0$ the roots of $\tilde{p}$ are

$$
t_{0}(0)=0, \quad\left(t_{j}(0)\right)^{\ell}=2 \ell \quad \forall j=1, \ldots, \ell .
$$

To be determined we take $t_{1}(0):=(2 \ell)^{1 / \ell}$ to be the positive real root of $2 \ell$ and the other roots in counterclockwise order. Correspondingly we will have

$$
E^{1 / \ell} t_{0}(\rho)=s_{m}(E, L), \quad E^{1 / \ell} t_{1}(\rho)=s_{M}(E, L) .
$$

Denote

$$
d:=\min _{j \neq l}\left|t_{j}(0)-t_{l}(0)\right|
$$

then there exists $\rho_{*}$ s.t., for $\rho<\rho_{*}$ one has

$$
\min _{j \neq l}\left|t_{j}(\rho)-t_{l}(\rho)\right| \geq d / 2
$$

and correspondingly

$$
\min _{j \neq l}\left|s_{j}(E, L)-s_{l}(E, L)\right| \geq \frac{d}{2} E^{\frac{1}{\ell}}
$$

The function to be integrated in $\mathrm{A} .5 \mathrm{~s}$

$$
F(z):=\frac{\sqrt{2}}{2 \pi} \frac{1}{z} \sqrt{-p(z, E, L)},
$$

In order to make it holomorphic we cut $\mathbb{C}$ along the segments $b_{j}$ joining $s_{2(j-1)}(E, L)$ with $s_{2 j-1}(E, L), j=1, \ldots,\left\lceil\frac{\ell}{2}\right\rceil$; if $\ell$ is odd, there is a last cut $b_{\left\lceil\frac{\ell}{2}\right\rceil}$, which is the half-line parallel to the real axis joining $s_{\ell}(E, L)$ with $\infty$. Remark that $b_{1}$ is the interval of integration in which we are interested.

We are now ready to choose the curve over we integrate to apply the method of the residue. To this end we define

$$
\mathrm{M}:=\max _{j=1, \ldots, \ell, \rho<\rho_{*}} \operatorname{Re}\left(t_{j}(\rho)\right)
$$




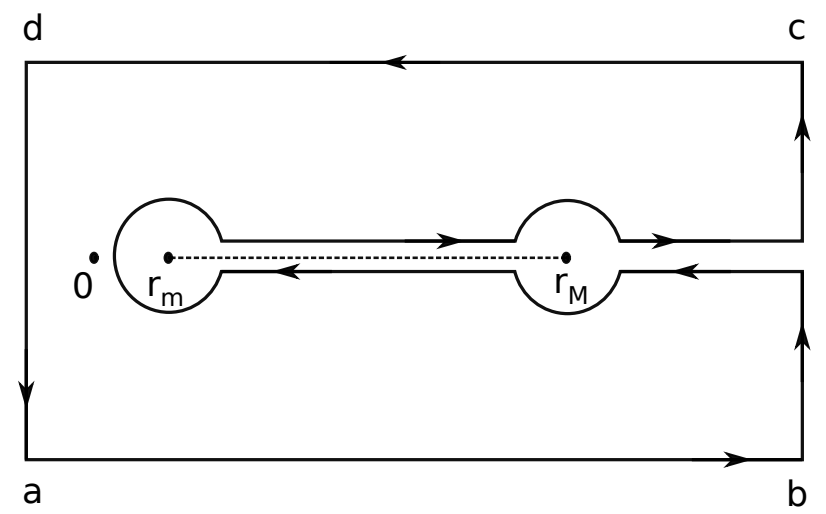

Figure 1: Representation of the path $\Gamma_{\varepsilon}$. The radius of the circles is $\varepsilon$, and the points $a, b, c, d$ are defined as in A.12.

and take the curve $\Gamma_{\varepsilon}$ described in Figure 1, with

$$
\begin{aligned}
& a=\left(-\frac{d}{4}-\mathrm{i} \frac{d}{4}\right) E^{\frac{1}{\ell}}, \quad b=\left(2 \mathrm{M}-\mathrm{i} \frac{d}{4}\right) E^{\frac{1}{\ell}}, \\
& c=\left(2 \mathrm{M}+\mathrm{i} \frac{d}{4}\right) E^{\frac{1}{\ell}}, \quad d=\left(-\frac{d}{4}+\mathrm{i} \frac{d}{4}\right) E^{\frac{1}{\ell}} .
\end{aligned}
$$

We also denote $\gamma_{R}$ the boundary of the rectangle $a b c d$. With this notation we have

$$
\begin{aligned}
2 a_{r} & =\lim _{\varepsilon \rightarrow 0} \int_{\Gamma_{\varepsilon}} F(z) d z-\int_{\gamma_{R}} F(z) d z \\
& =2 \pi \operatorname{iRes}(F, 0)-\int_{\gamma_{R}} F(z) d z=-|L|-\int_{\gamma_{R}} F(z) d z .
\end{aligned}
$$

To get the thesis just define

$$
f(E, L):=-\int_{\gamma_{R}} F(z) d z
$$

and remark that this is analytic in the considered domain.

Joining the results of these two lemmas one gets

Corollary A.3. There exists function $f=f(E, L)$ analytic in the domain

$$
\left\{(E, L)|E>0,| L \mid<\left(\frac{2 \ell}{\ell+1} E\right)^{\frac{\ell+1}{2 \ell}}\right\},
$$


such that

$$
a_{r}(E, L)=-\frac{1}{2}|L|+f(E, L) .
$$

We finally prove Lemma 4.5 :

Proof of Lemma 4.5. Let

$$
a_{1}:= \begin{cases}a_{r} & \text { if } L \geq 0 \\ a_{r}-L & \text { if } L<0 .\end{cases}
$$

then, by Corollary A.15, there exists a function $f$ analytic in the domain A.14 s.t.

$$
a_{1}=-\frac{L}{2}+f(E, L)
$$

so that $a_{1}$ itself is analytic in such a domain. This concludes the proof of Items (1) and (2).

We come to the homogeneity properties. Take $L \neq 0$, then by (4.4) and performing the change of variables $r=|L|^{\frac{1}{\ell+1}} x$ in 4.4 , one sees that

$$
a_{r}=\frac{\sqrt{2 E}}{\pi}|L|^{\frac{1}{\ell+1}} F(\rho), \quad F(\rho)=\int_{x_{m}}^{x_{M}} \sqrt{1-\rho\left(\frac{1}{2 x^{2}}+\frac{x^{2 \ell}}{2 \ell}\right)} d x
$$

with $\rho$ as in A.7) and $x_{m}, x_{M}$ solving the equation $\rho \frac{x^{2 \ell}}{2 \ell}+\rho \frac{1}{2 x^{2}}-1=0$. As a consequence, for any $\lambda>0 a_{r}$ satisfies

$$
a_{r}\left(\lambda^{\frac{2 \ell}{\ell+1}} E, \lambda L\right)=\lambda^{\frac{\ell}{\ell+1}} \sqrt{2 E} \lambda^{\frac{1}{\ell+1}}|L| F(\rho)=\lambda a_{r}(E, L),
$$

also $a_{1}$ defined as in A.16 satisfies

$$
a_{1}\left(\lambda^{\frac{2 \ell}{\ell+1}} E, \lambda L\right)=\lambda a_{r}(E, L) .
$$

Since $h_{0}$ and $a_{2}$ as in (4.2) are quasi-homogeneous functions of $x, \xi$, one immediately deduces the quasi-homogeneity of $a_{1}$. The homogeneity of $h_{0}$ as a function of $a$ also immediately follows from A.17). We still have to consider the case $L=0$. In this case the result immediately follows by continuity, by taking the limit of the functions to this set.

\section{B Proof of Lemma 5.12}

First we prove the following 
Lemma B.1. Let $\Pi^{*}:=a^{-1}\left(\Pi^{\circ}\right)$ and suppose $f \in C^{\infty}\left(\mathbb{R}^{2}\right)$ is such that $f \circ \phi_{a}^{\varphi}=f, \forall \varphi$ then there exists $\tilde{f} \in C^{\infty}\left(\Pi^{\circ}\right)$ s.t. $f=\tilde{f} \circ a$ on $\Pi^{*}$.

Proof. Introducing action angle coordinates $(a, \varphi)$, which by the standard theory (see e.g. Dui80]) are smooth and globally defined on the set $\Pi^{*}$, the function $f$ is a $C^{\infty}$ function of $(a, \varphi)$ which however does not depend on $\varphi$. This is the wanted function $\tilde{f}$.

Lemma B.2. There exist angle variables $\varphi$ that are quasi-homogeneous of degree 0 as functions on $\Pi^{*}$, namely they satisfy

$$
\varphi\left(\lambda x, \lambda^{\ell} \xi\right)=\varphi(x, \xi) \quad \forall(x, \xi) \in \Pi^{*}, \forall \lambda \in \mathbb{R}^{+} .
$$

Proof. Let $\varphi=\left(\varphi_{1}, \varphi_{2}\right): \Pi^{*} \rightarrow \mathbb{T}^{2}$ be such that $(a, \varphi)$ are global action angle coordinates. For any choice of $\bar{a} \in \Pi^{\circ}$ with $\|\bar{a}\|=1$, let $\left(x_{0}, \xi_{0}\right) \in \Pi^{*}$ be such that $a\left(x_{0}, \xi_{0}\right)=\bar{a}$ and $\varphi\left(x_{0}, \xi_{0}\right)=0$. Remark that it exists because the action $\phi_{a}^{\varphi}$ on the level surfaces of $a$ is transitive. Furthermore $\left(x_{0}, \xi_{0}\right)$ is a function of $a$ only. For $(x, \xi) \in \Pi^{*}$, define $\lambda \in \mathbb{R}^{+}$and $(\widetilde{x}, \widetilde{\xi})$ by

$$
(x, \xi)=\left(\lambda \tilde{x}, \lambda^{\ell} \tilde{\xi}\right), \quad \text { and } \quad\|a(\widetilde{x}, \widetilde{\xi})\|=1 .
$$

Observe that with this definition $\lambda$ is a function of the actions $a$ only. Then this implies that the function $\tilde{\varphi}=\left(\tilde{\varphi}_{1}, \tilde{\varphi}_{2}\right): \Pi^{*} \rightarrow \mathbb{T}^{2}$ given by

$$
\tilde{\varphi}(x, \xi):=\varphi(x, \xi)-\varphi\left(\lambda x_{0}, \lambda^{\ell} \xi_{0}\right)
$$

still defines angle coordinates conjugated to the actions $a$ on the set $\Pi^{*}$. This is due to the fact that $\varphi\left(\lambda x_{0}, \lambda^{\ell} \xi_{0}\right)$ is a function of the actions only. Remark also that

$$
\tilde{\varphi}\left(\lambda x_{0}, \lambda^{\ell} \xi_{0}\right)=0, \quad \forall \lambda>0 .
$$

From now on we use only the angles $\tilde{\varphi}$, so we omit the tildes.

We are now going to prove that these angles are homogeneous functions of degree 0 on $\Pi^{*}$.

To this aim, we define for $\mu>0$ and $j=1,2$

$$
\varphi_{\mu, j}(x, \xi):=\varphi_{j}\left(\mu x, \mu^{\ell} \xi\right)
$$

First of all, we observe that since $(a, \varphi)$ are canonically conjugated variables, one has $\left\{a_{j} ; \varphi_{i}\right\}=\delta_{i, j}$, so one has

$$
\left\{a_{i} ; \varphi_{\mu, j}\right\}(x, \xi)=\mu^{\ell} \partial_{x} a_{i}(x, \xi) \cdot \partial_{\xi} \varphi_{j}\left(\mu x, \mu^{\ell} \xi\right)-\mu \partial_{\xi} a_{i}(x, \xi) \cdot \partial_{x} \varphi_{j}\left(\mu x, \mu^{\ell} \xi\right)
$$


Since $a_{i}$ is quasi-homogeneous of degree $\ell+1, \forall(x, \xi) \in \Pi^{*}$ one has

$$
\begin{gathered}
\mu^{\ell} \partial_{x} a_{i}(x, \xi)=\partial_{x} a_{i}\left(\mu x, \mu^{\ell} \xi\right), \\
\mu \partial_{\xi} a_{i}(x, \xi)=\partial_{\xi} a_{i}\left(\mu x, \mu^{\ell} \xi\right),
\end{gathered}
$$

one also obtains

$$
\left\{a_{i} ; \varphi_{\mu, j}\right\}(x, \xi)=\left\{a_{i} ; \varphi_{j}\right\}\left(\mu x, \mu^{\ell} \xi\right) \equiv \delta_{i, j} .
$$

Thus, using action angle coordinates to compute Poisson Brackets, one has

$$
\left\{a_{j} ; \varphi_{\mu, j}\right\} \equiv \frac{\partial \varphi_{\mu, j}}{\partial \varphi_{j}}=1
$$

therefore there exist functions $f_{\mu, j}(a)$, depending on the actions only, such that

$$
\varphi_{\mu, j}-\varphi_{j}=f_{\mu, j}(a) .
$$

To prove that $f_{\mu, j}$ is identically zero, fix a value $\bar{a}$ of $a$, corresponding to some point $(\bar{x}, \bar{\xi}) \in \Pi^{*}$ define $\lambda:=\|\bar{a}\|^{\frac{1}{\ell+1}}$, then we have

$$
\begin{aligned}
f_{\mu, j}(a(\bar{x}, \bar{\xi})) & =f_{\mu, j}\left(\lambda^{\ell+1} a\left(\frac{\bar{x}}{\lambda}, \frac{\bar{\xi}}{\lambda^{\ell}}\right)\right)=f_{\mu, j}\left(\lambda^{\ell+1} a\left(x_{0}, \xi_{0}\right)\right) \\
& =f_{\mu, j}\left(a\left(\lambda x_{0}, \lambda^{\ell} \xi_{0}\right)\right)=\varphi_{\mu, j}\left(\lambda x_{0}, \lambda^{\ell} \xi_{0}\right)-\varphi_{j}\left(\lambda x_{0}, \lambda^{\ell} \xi_{0}\right)=0-0=0 .
\end{aligned}
$$

Corollary B.3. Let $\check{x}, \check{\xi}: \Pi^{\circ} \times \mathbb{T}^{2} \rightarrow \Pi^{*}$ be the functions expressing the Cartesian coordinates in terms of the action angle coordinates, namely s.t.

$$
(\check{x}, \check{\xi}) \circ(a, \varphi)=I d, \quad(a, \varphi) \circ(\check{x}, \check{\xi})=I d .
$$

Then the following holds:

$\check{x}\left(\lambda^{\ell+1} a, \varphi\right)=\lambda \check{x}(a, \varphi), \quad \check{\xi}\left(\lambda^{\ell+1} a, \varphi\right)=\lambda^{\ell} \check{\xi}(a, \varphi) \quad \forall \lambda \in \mathbb{R}^{+},(a, \varphi) \in \Pi^{\circ} \times \mathbb{T}^{2}$.

We now prove Lemma 5.12

Proof. By Lemma B.1, there exists $\tilde{f} \in C^{\infty}\left(\Pi^{\circ}\right)$ such that $f=\tilde{f} \circ a$. Passing to action angle variables on the set $\Pi^{*}$, one has

$$
\tilde{f}(a)=f(\check{x}(a, \varphi), \check{\xi}(a, \varphi))
$$

First we prove the estimates for $a \in \Pi^{\circ}$. Consider for example

$$
\partial_{a_{1}} \tilde{f}=\partial_{x} f \cdot \partial_{a_{1}} \check{x}+\partial_{\xi} f \cdot \partial_{a_{1}} \check{\xi}
$$


thus, using $f \in S_{A N, \delta}^{m}$, and the estimates

$$
\left|\partial_{a}^{\beta} \check{x}(a, \varphi)\right| \lesssim\langle a\rangle^{\frac{1}{\ell+1}-|\beta|}, \quad\left|\partial_{a}^{\beta} \check{\xi}(a, \varphi)\right| \lesssim\langle a\rangle^{\frac{1}{\ell+1}-|\beta|} .
$$

which follow from $($ B.4 $)$ we can estimate such a quantity. One gets for $a \in \Pi^{\circ}$

$$
\left|\partial_{a} \tilde{f}\right| \lesssim\langle a\rangle^{m-\delta_{1}-\frac{\ell}{\ell+1}}+\langle a\rangle^{m-\delta_{2}-\frac{1}{\ell+1}} \simeq\langle a\rangle^{m-\varsigma} .
$$

Iterating and studying the other derivatives, one also has that $\forall \alpha \in \mathbb{N}^{2}$, implies

$$
\left|\partial_{a}^{\alpha} \tilde{f}\right| \lesssim\langle a\rangle^{m-\varsigma|\alpha|},
$$

but just for $a \in \Pi^{\circ}$. To get a symbol defined on the whole of $\mathbb{R}^{2}$, we consider again the cones $\mathcal{V}$ and $\mathcal{C}$ and the cutoff function $\Psi$ supported in $\mathcal{V}$ and equal to one in $\mathcal{C}$, and just consider the function

$$
f_{c}(a):=\tilde{f}(a) \Psi(a)(1-\chi(\|a\|)),
$$

which has all the claimed properties.

\section{References}

[Bam96] Dario Bambusi. Exponential stability of breathers in Hamiltonian networks of weakly coupled oscillators. Nonlinearity, 9(2):433$457,1996$.

[BBM11] D. Bambusi, M. Berti, and E. Magistrelli. Degenerate KAM theory for partial differential equations. J. Differential Equations, 250(8):3379-3397, 2011.

[BF16] Larry M Bates and Francesco Fassò. No monodromy in the champagne bottle, or singularities of a superintegrable system. Journal of Geometric Mechanics, 8(4):375, 2016.

[BF17] Dario Bambusi and Alessandra Fusè. Nekhoroshev theorem for perturbations of the central motion. Regul. Chaotic Dyn., $22(1): 18-26,2017$.

[BFS18] Dario Bambusi, Alessandra Fusè, and Marco Sansottera. Exponential stability in the perturbed central force problem. Regul. Chaotic Dyn., 23(7-8):821-841, 2018.

[BLM20a] Dario Bambusi, Beatrice Langella, and Riccardo Montalto. Growth of Sobolev norms for unbounded perturbations of the Laplacian on flat tori. Preprint, arXiv:2012.02654, 2020. 
[BLM20b] Dario Bambusi, Beatrice Langella, and Riccardo Montalto. On the spectrum of the Schrödinger operator on $T^{d}$ : a normal form approach. Communications in Partial Differential Equations, 45:1-18, 2020.

[BLM20c] Dario Bambusi, Beatrice Langella, and Riccardo Montalto. Spectral asymptotics of all the eigenvalues of Schrödinger operators on flat tori. Preprint, arXiv:2007.07865, 2020.

[CdV80] Yves Colin de Verdière. Spectre conjoint d'opérateurs pseudodifférentiels qui commutent. II. Le cas intégrable. Math. Z., 171(1):51-73, 1980.

[Cha83a] Anne-Marie Charbonnel. Calcul fonctionnel a plusieurs variables pour des operateurs pseudodifferentiels dans $R^{n}$. Israël Journal of Mathematics, 45(1):69-89, 1983.

[Cha83b] Anne-Marie Charbonnel. Spectre conjoint d'opérateurs pseudodifférentiels qui commutent. Ann. Fac. Sci. Toulouse Math. (5), 5(2):109-147, 1983.

[Cha86] Anne-Marie Charbonnel. Localisation et developpment asymptotique des elements du spectre conjoint d'operateurs pseudodifferentiels qui commutent. Integral Equations and operator theory, 9(4):502-536, 1986.

[Dui80] J. J. Duistermaat. On global action-angle coordinates. Comm. Pure Appl. Math., 33(6):687-706, 1980.

[FK04] Jacques Féjoz and Laurent Kaczmarek. Sur le théorème de Bertrand (d'après Michael Herman). Ergodic Theory Dynam. Systems, 24(5):1583-1589, 2004.

[FKT90] Joel Feldman, Horst Knörrer, and Eugene Trubowitz. The perturbatively stable spectrum of a periodic Schrödinger operator. Invent. Math., 100(2):259-300, 1990.

[FKT91] Joel Feldman, Horst Knörrer, and Eugene Trubowitz. Perturbatively unstable eigenvalues of a periodic Schrödinger operator. Comment. Math. Helv., 66(4):557-579, 1991.

[Fri90] Leonid Friedlander. On the spectrum of the periodic problem for the Schrödinger operator. Comm. Partial Differential Equations, 15(11):1631-1647, 1990. 
[HR82a] B. Helffer and D. Robert. Asymptotique des niveaux d'énergie pour des hamiltoniens à un degré de liberté. Duke Math. J., 49(4):853-868, 1982.

[HR82b] B. Helffer and D. Robert. Propriétés asymptotiques du spectre d'opérateurs pseudodifférentiels sur $\mathbf{R}^{n}$. Comm. Partial Differential Equations, 7(7):795-882, 1982.

[HSVN07] M. Hitrik, J. Sjöstrand, and S. Vu Ngoc. Diophantine tori and spectral asymptotics for nonselfadjoint operators. American journal of mathematics, 129(1):105-182, 2007.

[Kar96] Yu. E. Karpeshina. Perturbation series for the Schrödinger operator with a periodic potential near planes of diffraction. Comm. Anal. Geom., 4(3):339-413, 1996.

[Par08] Leonid Parnovski. Bethe-Sommerfeld conjecture. Ann. Henri Poincaré, 9(3):457-508, 2008.

[PS10] Leonid Parnovski and Alexander V. Sobolev. Bethe-Sommerfeld conjecture for periodic operators with strong perturbations. Invent. Math., 181(3):467-540, 2010.

[PS12] Leonid Parnovski and Roman Shterenberg. Complete asymptotic expansion of the integrated density of states of multidimensional almost-periodic Schrödinger operators. Ann. of Math. (2), 176(2):1039-1096, 2012.

[Roy07] Nicolas Roy. A semi-classical K. A. M. theorem. Comm. Partial Differential Equations, 32(4-6):745-770, 2007.

[Rüs01] H. Rüssmann. Invariant tori in non-degenerate nearly integrable Hamiltonian systems. Regul. Chaotic Dyn., 6(2):119-204, 2001.

[Vel15] Oktay Veliev. Multidimensional periodic Schrödinger operator, volume 263 of Springer Tracts in Modern Physics. Springer, Cham, 2015. Perturbation theory and applications. 